\title{
EXTENDING HUPPERT'S CONJECTURE FROM NON-ABELIAN SIMPLE GROUPS TO QUASI-SIMPLE GROUPS
}

\author{
NGUYEN NGOC HUNG, PHILANI R. MAJOZI, \\ HUNG P. TONG-VIET AND THOMAS P. WAKEFIELD
}

\begin{abstract}
We propose to extend a conjecture of Bertram Huppert [Illinois J. Math. 44 (2000) 828-842] from finite non-Abelian simple groups to finite quasi-simple groups. Specifically, we conjecture that if a finite group $G$ and a finite quasi-simple group $H$ with $\operatorname{Mult}(H / \mathbf{Z}(H))$ cyclic have the same set of irreducible character degrees (not counting multiplicity), then $G$ is isomorphic to a central product of $H$ and an Abelian group. We present a pattern to approach this extended conjecture and, as a demonstration, we confirm it for the special linear groups in dimensions 2 and 3.
\end{abstract}

\section{Introduction}

It is well known that the character degree $\operatorname{set} \operatorname{cd}(G)$ of a finite group $G$ has a strong influence on the structure of $G$. While $\operatorname{cd}(G)$ does not completely determine the structure of $G$ in general, non-Abelian simple groups seem to have a very close connection with their character degrees. Indeed, in the late 1990s, Huppert [9] put forward the following, which predicted that every finite non-Abelian simple group is essentially determined by its character degrees.

Received November 9, 2016.

N. N. Hung is partially supported by the NSA Young Investigator Grant \#H9823014-1-0293 and a Faculty Scholarship Award from Buchtel College of Arts and Sciences, The University of Akron. Tong-Viet's work is based on the research supported wholly by the National Research Foundation of South Africa (Grant Number 93408). P. R. Majozi's work was supported by the School of Mathematics, Statistics and Computer Science, The University of KwaZulu-Natal.

2010 Mathematics Subject Classification. Primary 20C15. Secondary 20C33, 20C34, $20 \mathrm{C} 30$. 
Conjecture 1.1 (Huppert's conjecture). Let $S$ be a finite non-Abelian simple group and $G$ be a finite group. Then $\operatorname{cd}(G)=\operatorname{cd}(S)$ if and only if $G \cong S \times A$ for an Abelian group $A$.

Though Huppert's conjecture is still open, there has been much progress on the verification of the conjecture for several families of simple groups, especially the simple groups of exceptional Lie type and simple classical groups in low dimension, see [9], [23], [24], [25], [26], [27], [28], [29].

Recently, interesting examples have been found [18], [19] showing that the character degree set does not determine the solvability of the group. That is, if $\operatorname{cd}(G)=\operatorname{cd}(H)$ and $H$ is solvable, then $G$ need not be solvable. In fact, the group $G$ in these examples can be chosen to be perfect, and therefore Conjecture 1.1 cannot not be generalized to arbitrary perfect groups. Can it be extended at least to quasi-simple groups?

It has been recently shown in [1], [22] that if a finite group $G$ and a quasi-simple group $H$ have isomorphic complex group algebras, then $G \cong H$. Equivalently, the set of character degrees counting multiplicities of a finite quasi-simple group uniquely determines the group. Inspired by this result, we propose to extend Huppert's conjecture from non-Abelian simple groups to quasi-simple groups as follows. Here and from now on, the Schur multiplier of a group $X$ is denoted by $\operatorname{Mult}(X)$.

CONJECTURE 1.2. Let $G$ be a finite group and $H$ a finite quasi-simple group with $\operatorname{Mult}(H / \mathbf{Z}(H))$ cyclic. Then $\operatorname{cd}(G)=\operatorname{cd}(H)$ if and only if $G \cong H \circ A$, a central product of $H$ and an Abelian group $A$.

The condition on the Schur multiplier of $H / \mathbf{Z}(H)$ is essential and we would like to thank the referee for bringing this to our attention. For example, consider $G=2^{2} \cdot \Omega_{8}^{+}(2)$ and $H=G / Z$ for $Z$ a central subgroup of $G$ of order 2 . Since $G$ has no faithful irreducible representations, we have $\operatorname{cd}(G)=\operatorname{cd}(H)$, but $G$ is not isomorphic to a central product of $H$ with any Abelian group. Note that most of the finite simple groups have cyclic Schur multipliers, except the orthogonal groups $P \Omega_{2 n}^{+}(q)$ with $n$ even and some "small" groups of Lie type which can be found in [5]. To make the conjecture valid for all quasisimple groups, we propose the following: Let $G$ be a finite group and $H$ a finite quasi-simple group. Then $\operatorname{cd}(G)=\operatorname{cd}(H)$ if and only if $G \cong K \circ A$, where $K$ is a cover of $H / \mathbf{Z}(H)$ such that $\operatorname{cd}(K)=\operatorname{cd}(H)$ and $A$ is an Abelian group.

Recall that a group $G$ is said to be a central product of two groups $H$ and $A$ (over $Z$ ) if $Z=H \cap A,[H, A]=1$, and $G=H A$. For any $\lambda \in \operatorname{Irr}(Z)$, there is then a bijective correspondence $\operatorname{Irr}(H \mid \lambda) \times \operatorname{Irr}(A \mid \lambda) \rightarrow \operatorname{Irr}(G \mid \lambda)$ such that if $(\alpha, \beta) \mapsto \chi$ then $\chi(1)=\alpha(1) \beta(1)$. (Here $\operatorname{Irr}(G \mid \lambda)$ denotes the set of irreducible characters of $G$ lying above $\lambda$.) Therefore, when $A$ is Abelian, we have $\operatorname{cd}(H \circ A)=\operatorname{cd}(H)$ and thus the "if" implication of the conjecture follows. The real work on the conjecture is therefore the "only if" implication. 
Huppert outlined in [9] a pattern consisting of five steps to attack his conjecture, and this pattern and variants thereof have been successfully used in verifying the conjecture for several non-Abelian simple groups. Drawing upon his method, we propose the following pattern to approach Conjecture 1.2. In the following, assume that $\operatorname{cd}(G)=\operatorname{cd}(H)$, where $H$ is a finite quasi-simple group.

Step 1: Show $G^{\prime}=G^{\prime \prime}$.

Step 2: Suppose that $G^{\prime} / M$ is a chief factor of $G$. Then $G^{\prime} / M \cong S^{k}$, where $S$ is a non-Abelian simple group and $k \geq 1$ is an integer. Show $G^{\prime} / M \cong$ $H / \mathbf{Z}(H)$.

Step 3: Show that $G^{\prime}$ is isomorphic to a perfect central cover of $H / \mathbf{Z}(H)$.

Step 4: Show that $G=G^{\prime} \circ \mathbf{C}_{G}\left(G^{\prime}\right)$ and $\mathbf{C}_{G}\left(G^{\prime}\right)$ is Abelian. In particular, $\operatorname{cd}(G)=\operatorname{cd}\left(G^{\prime}\right)$, and thus $\operatorname{cd}\left(G^{\prime}\right)=\operatorname{cd}(H)$.

Step 5: Show that the perfect central covers of $H / \mathbf{Z}(H)$ have distinct sets of character degrees. Together with Steps 3 and 4 , we have $H \cong G^{\prime}$, and it follows that $G \cong H \circ \mathbf{C}_{G}\left(G^{\prime}\right)$.

It is worth commenting that Steps 1, 2, and 4 in our pattern are similar and correspond to Steps 1, 2, and 5, respectively, in Huppert's pattern; while Steps 3 and 5 here are new and needed to work with quasi-simple groups. As a demonstration, we confirm Conjecture 1.2 for the special linear groups in dimensions 2 and 3 .

TheOREM 1.3. Let $G$ be a finite group and let $H \cong \mathrm{SL}_{2}(q)$ with $q \geq 5$ or $H \cong \mathrm{SL}_{3}(q)$ with $q \geq 2$. Then $\operatorname{cd}(G)=\operatorname{cd}(H)$ if and only if $G \cong H \circ A$, a central product of $H$ and an Abelian group $A$.

We could have followed the above method to prove the theorem for $\operatorname{SL}_{2}(q)$ but this is rather long. Instead we utilize the notion of prime graphs of finite groups and provide a much shorter proof, see Section 3. Recall that the prime graph $\Delta(G)$ of a finite group $G$ is a graph whose vertices are the primes dividing at least one character degree in $\operatorname{cd}(G)$, and two distinct vertices $r, s$ are joined if and only if the product $r s$ divides some character degree of $G$. Observe that $\Delta\left(\mathrm{SL}_{2}(q)\right)$ with $q \geq 5$ is disconnected. It follows that $\Delta(G)$ is disconnected whenever $\operatorname{cd}(G)=\operatorname{cd}\left(\mathrm{SL}_{2}(q)\right)$ and thus we can apply the structure theorem for these groups due to Lewis and White in [13]. This prime-graph method however does not work for $\mathrm{SL}_{3}(q)$ since the prime graph of this group is connected when $q \geq 4$.

\section{Some preliminary lemmas}

For the reader's convenience, we collect and prove in this section some preliminary results which will be useful for the proof of Theorem 1.3. We begin with a known fact on characters of a central product, see [11, Lemma 5.1], for instance. 
LEMmA 2.1. Let $G=H \circ K$ be a central product with $Z=H \cap K$ and let $\lambda$ be a linear character of $Z$. Given any character $\alpha \in \operatorname{Irr}(H \mid \lambda)$ and $\beta \in \operatorname{Irr}(K \mid \lambda)$, there exists a unique character $\chi \in \operatorname{Irr}(G \mid \lambda)$ such that $\chi_{H}$ and $\chi_{K}$ are multiples of $\alpha$ and $\beta$, respectively. Furthermore, for every $h \in H$ and $k \in K$, we have $\chi(h k)=\alpha(h) \beta(k)$, and every irreducible character of $G$ arises in this way.

The next technical lemma will be needed in Step 1.

LEMMA 2.2 (Lemma 2.3 of [25]). Let $G / N$ be a solvable factor group of $G$, minimal with respect to being non-Abelian. Then two cases can occur:

(a) $G / N$ is an $r$-group for some prime $r$. Hence there exists $\psi \in \operatorname{Irr}(G / N)$ such that $\psi(1)=r^{b}>1$ for some prime $r$. If $\chi \in \operatorname{Irr}(G)$ and $r \nmid \chi(1)$, then $\chi \tau \in \operatorname{Irr}(G)$ for all $\tau \in \operatorname{Irr}(G / N)$.

(b) $G / N$ is a Frobenius group with an elementary Abelian Frobenius kernel $F / N$. Then $l=|G: F| \in \operatorname{cd}(G),|F: N|=r^{c}$ for some prime $r$, and $c$ is the smallest integer such that $r^{c}-1 \equiv 0(\bmod l)$. Also, for every $\psi \in \operatorname{Irr}(F)$, either $|G: F| \psi(1) \in \operatorname{cd}(G)$ or $|F: N| \mid \psi(1)^{2}$. Moreover,

(i) If no proper multiple of $l$ is in $\operatorname{cd}(G)$, then $\chi(1) \mid l$ for all $\chi \in \operatorname{Irr}(G)$ such that $r \nmid \chi(1)$, and if $\chi \in \operatorname{Irr}(G)$ such that $\chi(1) \nmid l$, then $r^{c} \mid \chi(1)^{2}$.

(ii) If $\chi \in \operatorname{Irr}(G)$ such that no proper multiple of $\chi(1)$ is in $\operatorname{cd}(G)$, then either $l$ divides $\chi(1)$ or $r^{c}$ divides $\chi(1)^{2}$. Moreover, if $\chi(1)$ is divisible by no non-trivial proper character degree in $G$, then $l=\chi(1)$ or $r^{c}$ $\chi(1)^{2}$.

The following two results are Lemmas 2 and 3 in [9].

LEMMA 2.3. Let $N \unlhd G$ and $\chi \in \operatorname{Irr}(G)$.

(a) If $\chi_{N}=\theta_{1}+\theta_{2}+\cdots+\theta_{k}$ with $\theta_{i} \in \operatorname{Irr}(N)$, then $k$ divides $|G: N|$. In particular, if $\chi(1)$ is relatively prime to $|G: N|$, then $\chi_{N} \in \operatorname{Irr}(N)$.

(b) (Gallagher) If $\chi_{N} \in \operatorname{Irr}(N)$, then $\chi \theta \in \operatorname{Irr}(G)$ for every $\theta \in \operatorname{Irr}(G / N)$.

Lemma 2.4. Suppose $N \unlhd G$ and $\vartheta \in \operatorname{Irr}(N)$. By $I=I_{G}(\vartheta)$ we denote the inertia subgroup of $\vartheta$ in $G$.

(a) If $\vartheta^{I}=\sum_{i=1}^{k} \varphi_{i}$ with $\varphi_{i} \in \operatorname{Irr}(I)$, then $\varphi_{i}^{G} \in \operatorname{Irr}(G)$. In particular $\varphi_{i}(1) \mid G$ : $I \mid \in \operatorname{cd}(G)$.

(b) If $\vartheta$ allows an extension $\vartheta_{0}$ to $I$, then $\left(\vartheta_{0} \tau\right)^{G} \in \operatorname{Irr}(G)$ for all $\tau \in$ $\operatorname{Irr}(I / N)$. In particular $\vartheta(1) \tau(1)|G: I| \in \operatorname{cd}(G)$.

(c) If $\varrho \in \operatorname{Irr}(I)$ such that $\varrho_{N}=e \vartheta, \varrho=\vartheta_{0} \tau_{0}$, where $\vartheta_{0}$ is a character of an irreducible projective representation of $I$ of degree $\vartheta(1)$ while $\tau_{0}$ is the character of an irreducible projective representation of $I / N$ of degree $e$.

The next lemma will be used to verify Steps 2 and 3.

LEMMA 2.5 ([2, Lemma 5$])$. Let $N$ be a non-Abelian minimal normal subgroup of $G$ so that $N \cong S^{k}$, where $S$ is a non-Abelian simple group and $k \geq 1$ is an integer. If $\sigma \in \operatorname{Irr}(S)$ extends to $\operatorname{Aut}(S)$, then $\sigma^{k} \in \operatorname{Irr}(N)$ extends to $G$. 
We shall combine Lemma 2.5 with the following result to prove Step 2.

LEMma 2.6. Let $S$ be a non-Abelian simple group. The following holds.

(a) If $S$ is an alternating group $\mathrm{A}_{n}$ with $n \geq 7$, then $\operatorname{Irr}(S)$ contains at least five non-linear irreducible characters of different degrees which extend to Aut $\left(\mathrm{A}_{n}\right) ;$ moreover, $S$ has two consecutive characters of degrees $n(n-3) / 2$ and $(n-1)(n-2) / 2$ that both extend to $\operatorname{Aut}(S)$.

(b) If $S$ is a sporadic simple group or the Tits group then $S$ has at least five distinct non-linear irreducible characters of different degrees which extend to $\operatorname{Aut}(S)$. Moreover, $S$ has two non-linear irreducible characters of relatively prime degrees which both extend to $\operatorname{Aut}(S)$.

(c) If $S$ is a simple group of Lie type in characteristic p, then the Steinberg character $\mathrm{St}_{S}$ of $S$ of degree $|S|_{p}$ extends to $\operatorname{Aut}(S)$.

Proof. If $S=\mathrm{A}_{7}$, the Tits group or a sporadic simple group, then $\operatorname{Irr}(S)$ contains at least five non-linear irreducible characters of different degrees which extend to $\operatorname{Aut}(S)$ by inspecting [5]. Now suppose that $S=\mathrm{A}_{n}$ with $n \geq 8$. Then the irreducible characters of $\mathrm{S}_{n}=\operatorname{Aut}\left(\mathrm{A}_{n}\right)$ labelled by the partitions

$$
(n-1,1),\left(n-2,1^{2}\right),(n-2,2),\left(n-3,1^{3}\right),(n-3,3)
$$

are all irreducible upon restriction to $\mathrm{A}_{n}$ and have distinct non-trivial degrees. So, $\operatorname{Irr}\left(\mathrm{A}_{n}\right)$ contains at least five non-linear irreducible characters of different degrees which extend to $\operatorname{Aut}(S)$. Other statements can be found in [2].

The next results will be used to verify Step 3.

LEMma 2.7 ([9, Lemma 3]). Let $N \unlhd G$ and let $\theta \in \operatorname{Irr}(N)$ be $G$-invariant. If $\phi \in \operatorname{Irr}(G)$ lies above $\theta$ then $\phi=\theta_{0} \tau$, where $\theta_{0}$ is the character of an irreducible projective representation of $G$ of degree $\theta(1)$ and $\tau$ is the character of an irreducible projective representation of $G / N$.

LEMmA 2.8 ([15, Theorem 2.3]). Let $N$ be a normal subgroup of a finite group $G$ and let $\theta \in \operatorname{Irr}(N)$ be $G$-invariant. If $\chi(1) / \theta(1)$ is a power of a fixed prime $p$ for every $\chi \in \operatorname{Irr}(G \mid \theta)$ then $G / N$ is solvable.

Recall that $\operatorname{Mult}(G)$ denotes the Schur multiplier of $G$. A group $H$ is called a covering group (or a cover for short) of $G$ if there exists a subgroup $A$ of $H$ such that $A \leq H^{\prime} \cap \mathbf{Z}(H)$ and $H / A \cong G$. A covering group $H$ of $G$ is called a universal cover or a Schur cover if $|\mathbf{Z}(H)|=|\operatorname{Mult}(G)|$.

We would like to take this opportunity to correct an inaccuracy in $[21$, Lemma 7.3]. It should be as follows.

LEMMA 2.9. Let $S$ be a non-Abelian simple group. Let $A$ be an Abelian group such that $|A| \leq|\operatorname{Mult}(S)|$. We have

(a) if $S$ is not $\operatorname{PSL}_{3}(4)$ and $\operatorname{PSU}_{4}(3)$ then $|S|>|\operatorname{Aut}(A)|$,

(b) if $S=\mathrm{PSU}_{4}(3)$ and $|A||| \operatorname{Mult}(S) \mid$ then $|S|>|\operatorname{Aut}(A)|$, and 
(c) if $S=\mathrm{PSL}_{3}(4)$ and $|A||| \operatorname{Mult}(S) \mid$ then any perfect cover of $S$ cannot be imbedded into $\operatorname{Aut}(A)$.

In particular, for any $S$, if $|A|$ divides $|\operatorname{Mult}(S)|$ then any perfect cover of $S$ cannot be imbedded into $\operatorname{Aut}(A)$.

Proof. When $S$ is not $\mathrm{PSL}_{3}(4)$ and $\mathrm{PSU}_{4}(3)$ then the proof follows as in [21, Lemma 7.3]. Recall that $\operatorname{Mult}\left(\mathrm{PSU}_{4}(3)\right)=C_{3} \times C_{3} \times C_{4}$ is of order 36. So if $|A|$ divides $\left|\operatorname{Mult}\left(\mathrm{PSU}_{4}(3)\right)\right|$ then the maximal value of $|\operatorname{Aut}(A)|$ is $\left|\mathrm{GL}_{2}(3)\right|\left|\mathrm{GL}_{2}(2)\right|$, which is smaller than $\left|\mathrm{PSU}_{4}(3)\right|$. Finally, recall that Mult $\left(\mathrm{PSL}_{4}(3)\right)=C_{4} \times C_{4} \times C_{3}$ is of order 48 . It is easy to see that if $|A| \mid 48$ and $|\operatorname{Aut}(A)| \geq\left|\mathrm{PSL}_{4}(3)\right|$ then $A \cong C_{2}^{4}$ or $C_{2}^{4} \times C_{3}$, and in either case, any perfect cover of $\mathrm{PSL}_{4}(3)$ cannot be embedded into $\operatorname{Aut}(A)$.

The inaccuracy in [21, Lemma 7.3] fortunately does not affect the main results there. One just replaces the hypothesis $|M| \leq|\operatorname{Mult}(S)|$ in Lemmas 7.4, 7.5, and 7.6 by $|M||| \operatorname{Mult}(S) \mid$ and argues as before. Now we apply Lemma 2.9 to have the following result, which is essential in Step 3.

LEMMA 2.10. Let $H$ be a quasi-simple group, $G$ be a perfect group, and $M$ a normal subgroup of $G$ such that $G / M \cong H$ and $\left|M: M^{\prime}\right|$ divides $|\operatorname{Mult}(H / \mathbf{Z}(H))|$. Then $G / M^{\prime}$ is isomorphic to a perfect cover of $H / \mathbf{Z}(H)$.

Proof. As $M / M^{\prime}$ is Abelian and normal in $G / M^{\prime}$, we have

$$
M / M^{\prime} \leq \mathbf{C}_{G / M^{\prime}}\left(M / M^{\prime}\right) \unlhd G / M^{\prime} .
$$

We first consider the case $\mathbf{C}_{G / M^{\prime}}\left(M / M^{\prime}\right)=G / M^{\prime}$. Then $M / M^{\prime}$ is central in $G / M^{\prime}$ and we deduce that $G / M^{\prime}$ is a perfect cover of $G / M \cong H$, which implies that $G / M^{\prime}$ is a perfect cover of $H / \mathbf{Z}(H)$, as wanted.

The lemma is proved if we can show that $\mathbf{C}_{G / M^{\prime}}\left(M / M^{\prime}\right)$ cannot be a proper normal subgroup of $G / M^{\prime}$. Assume so, then

$$
\frac{\mathbf{C}_{G / M^{\prime}}\left(M / M^{\prime}\right)}{M / M^{\prime}} \triangleleft \frac{G / M^{\prime}}{M / M^{\prime}} \cong G / M \cong H .
$$

Therefore,

$$
\frac{\mathbf{C}_{G / M^{\prime}}\left(M / M^{\prime}\right)}{M / M^{\prime}} \leq \mathbf{Z}(G / M) \cong \mathbf{Z}(H)
$$

and hence

$$
\frac{G / M^{\prime}}{\mathbf{C}_{G / M^{\prime}}\left(M / M^{\prime}\right)} \cong \frac{G / M}{\mathbf{C}_{G / M^{\prime}}\left(M / M^{\prime}\right) /\left(M / M^{\prime}\right)}
$$

is a perfect cover of $H / \mathbf{Z}(H)$. But the factor group on the left-hand side can be embedded into $\operatorname{Aut}\left(M / M^{\prime}\right)$ and this violates Lemma 2.9. 


\section{Proof of Theorem 1.3 for $\mathrm{SL}_{2}(q)$}

For a finite group $G$, let $\rho(G)$ denote the set of prime divisors of the character degrees of $G$. The prime graph $\Delta(G)$ of $G$ is the graph whose vertex set is $\rho(G)$ and there is an edge between two distinct primes $p$ and $q$ if and only if the product $p q$ divides some character degree of $G$.

From now on, we write $\pi(n)$ to denote the set of prime divisors of a positive integer $n$.

We now prove Theorem 1.3 for the linear groups $\mathrm{SL}_{2}(q)$.

THEOREM 3.1. Let $G$ be a finite group and let $q=p^{f} \geq 5$ be a power of a prime $p$. Then $\operatorname{cd}(G)=\operatorname{cd}\left(\mathrm{SL}_{2}(q)\right)$ if and only if $G \cong \mathrm{SL}_{2}(q) \circ A$, where $A$ is Abelian.

Proof. In view of Theorem 2 in [9], we can assume that $q \geq 5$ is odd. In this case, recall that

$$
\operatorname{cd}\left(\mathrm{SL}_{2}(q)\right)=\left\{1, \frac{1}{2}(q \pm 1), q, q \pm 1\right\}
$$

and the prime graph $\Delta\left(\mathrm{SL}_{2}(q)\right)$ of $\mathrm{SL}_{2}(q)$ is disconnected with two connected components, namely $\{p\}$ and $\pi\left(q^{2}-1\right)$.

(1) $G^{\prime}=G^{\prime \prime}$.

Assume, to the contrary, that $G^{\prime} \neq G^{\prime \prime}$. Then we can find a solvable factor group $G / N$, minimal with respect to being non-Abelian. By Lemma 2.2, $G / N$ is an $r$-group for some prime $r$ or a Frobenius group.

Consider the possibility that $G / N$ is an $r$-group for some prime $r$. Choose $\chi \in \operatorname{Irr}(G)$ such that $\chi(1)$ is coprime to $r$, and furthermore $\chi(1)=q$ or $q+1$. Then $\chi_{N} \in \operatorname{Irr}(N)$ and hence $\chi \tau \in \operatorname{Irr}(G)$ for every $\tau \in \operatorname{Irr}(G / N)$. This is impossible since a proper multiple of $q$ or $q+1$ cannot be a character degree of $\mathrm{SL}_{2}(q)$.

We may now assume that $G / N$ is a Frobenius group with elementary Abelian Frobenius kernel $F / N$. Then $|G: F| \in \operatorname{cd}(G)$ and $|F: N|=r^{c}$ for some prime $r$ and a positive integer $c$. Furthermore, $c$ is the smallest positive integer such that $|G: F| \mid\left(r^{c}-1\right)$. Assume first that $r \neq p$. In Lemma 2.2(b)(ii), by choosing $\chi \in \operatorname{Irr}(G)$ to be a character of degree $q$, we deduce that $|G: F| \mid q$ and thus $|G: F|=q$. It follows that, for every $\varphi \in G$ of degree $q \pm 1, \varphi_{F} \in \operatorname{Irr}(F)$. Therefore, Lemma 2.2(b) implies that $r^{c} \mid(q \pm 1)^{2}$, which in turns implies that $r^{c} \mid 4 q$. As $q \mid\left(r^{c}-1\right)$, we then have $r^{c} \mid 4$, which implies that $q \leq 3$, a contradiction.

It remains to assume that $r=p$. Using Lemma 2.2(ii)(b) again, we have $|G: F|$ divides $q \pm 1$, which implies that $|G: F|=2$. This is possible only if $q=5$. But then, in Lemma 2.2(ii)(b), by taking $\chi \in \operatorname{Irr}(G)$ of degree 3, we again get a contradiction.

(2) $G^{\prime} \cong \mathrm{SL}_{2}(q)$ or $\mathrm{PSL}_{2}(q)$. 
Since $G^{\prime}$ is perfect, it is the smallest normal subgroup of $G$ such that $G / G^{\prime}$ is solvable and hence by [13, Theorem 6.3], $G^{\prime} / N \cong \mathrm{PSL}_{2}\left(q_{1}\right)$ for some prime power $q_{1}=p_{1}^{f_{1}}$, where $p_{1}$ is a prime, $C / N \subseteq \mathbf{Z}(G / N)$ with $C / N=$ $\mathbf{C}_{G / N}\left(G^{\prime} / N\right) ; p \nmid\left|G: C G^{\prime}\right|$; and if $N>1$, then either $G^{\prime} \cong \mathrm{SL}_{2}\left(q_{1}\right)$ or there is a normal subgroup $L$ of $G$ such that $G^{\prime} / L \cong \mathrm{SL}_{2}\left(q_{1}\right), L$ is elementary Abelian of order $q_{1}^{2}$, and $G^{\prime} / L$ acts transitively on the nonprincipal characters in $\operatorname{Irr}(L)$. Moreover, the connected components of $\Delta(G)$ are $\left\{p_{1}\right\}$ and $\pi\left(\left|G: C G^{\prime}\right|\right) \cup$ $\pi\left(q_{1}^{2}-1\right)$. Clearly, by comparing the sizes of the connected components, we see that $p=p_{1}$. Moreover, it is well known that the Steinberg character of $G^{\prime} / N \cong \mathrm{PSL}_{2}\left(q_{1}\right)$ of degree $q_{1}$ is extendible to $G$ and hence, $G$ has an irreducible character of degree $q_{1}=p^{f_{1}}>1$, from which it follows that $q_{1}=q$ as $\operatorname{cd}(G)=\operatorname{cd}\left(\mathrm{SL}_{2}(q)\right)$ and $q$ is the only non-trivial $p$-power degree in $\operatorname{cd}(G)$. If $G^{\prime} \cong \mathrm{SL}_{2}(q)$ or $\operatorname{PSL}_{2}(q)$, then we are done, so one can assume that $N$ is elementary Abelian of order $q^{2}$. Since $G^{\prime} / L$ acts transitively on $\operatorname{Irr}(L) \backslash\left\{1_{L}\right\}$, it is easy to deduce that $G^{\prime}$ has an irreducible character of degree $q^{2}-1$. Since $G^{\prime} \unlhd G$, every character degree of $G^{\prime}$ divides some character degree of $G$ and thus $q^{2}-1$ must divide some character degree in $\operatorname{cd}(G)$, which is impossible as $q \geq 5$.

(3) $G / C \cong \mathrm{PSL}_{2}(q)$ or $\mathrm{PGL}_{2}(q)$.

Let $G_{1} \unlhd G$ such that $G_{1} / C=(G / C) \cap \mathrm{PGL}_{2}(q)$. By [30, Theorem A], we have $\left|G: G_{1}\right|(q-1) \in \operatorname{cd}(G)$. Since $q \geq 5$, we must have $\left|G: G_{1}\right|(q-1)=q-1$ and thus $G=G_{1}$. Hence, $\operatorname{PSL}_{2}(q) \unlhd G / C \leq \mathrm{PGL}_{2}(q)$. Now the claim follows as $\mathrm{PSL}_{2}(q)$ has index 2 in $\mathrm{PGL}_{2}(q)$.

(4) $G^{\prime} \cong \mathrm{SL}_{2}(q)$.

Suppose to the contrary that $G^{\prime} \cong \mathrm{PSL}_{2}(q)$. Let $M=G^{\prime} C \unlhd G$. Then $M=G^{\prime} \times C$ as $G^{\prime} \cap C=1$. Notice that $C$ is central in $G$ since $N=1$ in this case. Now if $G / C \cong \mathrm{PSL}_{2}(q)$, then $G=M=G^{\prime} \times C$ and thus $\operatorname{cd}(G)=\operatorname{cd}\left(G^{\prime}\right)$ which implies that $\operatorname{cd}\left(\mathrm{SL}_{2}(q)\right)=\mathrm{cd}\left(\mathrm{PSL}_{2}(q)\right)$, a contradiction. Thus by (3), we have $G / C \cong \mathrm{PGL}_{2}(q)$ and hence $|G: M|=2$.

Let $\chi \in \operatorname{Irr}(G)$ with $\chi(1)=(q-\varepsilon) / 2 \geq 2$ where $q \equiv \varepsilon(\bmod 4)$. Let $\phi \in$ $\operatorname{Irr}(M)$ be an irreducible constituent of $\chi_{M}$. Then $\phi=\psi \times \lambda$, where $\psi \in \operatorname{Irr}\left(G^{\prime}\right)$ and $\lambda \in \operatorname{Irr}(C)$. Notice that $C$ is Abelian and $G^{\prime}$ is simple. As $(q+\varepsilon) / 2$ is the smallest non-trivial degree of $G^{\prime}$, we deduce that $\psi=1_{G^{\prime}}$, but then $\phi=\psi \times \lambda$ is a linear $G$-invariant character and since $G / M \cong C_{2}$ is cyclic, $\phi$ extends to $G$, so $\chi \in \operatorname{Irr}(G \mid \phi)$ must be linear by Gallagher's theorem [10, Corollary 6.17], a contradiction.

We can also choose $\chi \in \operatorname{Irr}(G)$ with $\chi(1)=(q+\varepsilon) / 2$. Then $\operatorname{gcd}(\chi(1), \mid G$ : $M \mid)=1$ and hence $\chi_{M} \in \operatorname{Irr}(M)$. In particular, $\chi_{M}$ is $G$-invariant. Since $M=G^{\prime} \times C$, where $C$ is central in $G$, we deduce that $\chi_{M}=\psi \times \lambda$ with $\psi \in \operatorname{Irr}\left(G^{\prime}\right)$ and $\lambda \in \operatorname{Irr}(C)$. Then $\psi(1)=\chi(1)=(q+\varepsilon) / 2$ and we know that every irreducible character of $G^{\prime}$ of degree $(q+\varepsilon) / 2$ is not invariant under the diagonal automorphism and $\lambda$ is $G$-invariant so that $I_{G}\left(\chi_{M}\right)=M<G$, which is a contradiction. 
(5) $G \cong G^{\prime} \circ C$ and $C$ is Abelian.

Since $G^{\prime} / N \cong \operatorname{PSL}_{2}(q)$ is non-Abelian simple, we deduce that $G^{\prime} \cap C=N$ and $N=\mathbf{Z}\left(G^{\prime}\right) \unlhd G$, and hence $N \subseteq \mathbf{Z}(G)$ as $|N|=2$. Since $G^{\prime}$ is perfect, by the Three Subgroups Lemma, we obtain that $\left[G^{\prime}, C\right]=1$, and so $G^{\prime} C=G^{\prime} \circ C$ is a central product.

Assume that $G / C \cong \mathrm{PGL}_{2}(q)$. Let $M=G^{\prime} C \unlhd G$ be a normal subgroup of index 2. Let $\chi \in \operatorname{Irr}(G)$ with $\chi(1)=(q+\varepsilon) / 2$. Then $\phi=\chi_{M} \in \operatorname{Irr}(M)=$ $\operatorname{Irr}\left(G^{\prime} \circ C\right)$ since $\operatorname{gcd}(\chi(1),|G: M|)=1$. Let $\phi_{N}=\phi(1) \lambda=\chi(1) \lambda$ with $\lambda \in$ $\operatorname{Irr}(N)$. If $\lambda \neq 1_{N}$, then $\phi \in \operatorname{Irr}\left(G^{\prime} \circ C \mid \lambda\right)$ and so by Lemma $2.1, \phi=\theta \cdot \varphi$ where $\theta \in \operatorname{Irr}\left(G^{\prime} \mid \lambda\right)$ and $\varphi \in \operatorname{Irr}(C \mid \lambda)$. Notice that the degrees of faithful irreducible characters of $G^{\prime} \cong \mathrm{SL}_{2}(q)$ are $(q-\varepsilon) / 2, q-1$ or $q+1$. Then $\theta(1) \in$ $\{(q-\varepsilon) / 2, q \pm 1\}$. Since $\phi(1)=\theta(1) \varphi(1)=(q+\varepsilon) / 2$, we see that $\theta(1)$ must divide $(q+\varepsilon) / 2$, which is impossible. Thus, $\lambda=1_{N}$ and so $\phi \in \operatorname{Irr}(M / N)=$ $\operatorname{Irr}\left(G^{\prime} / N \times C / N\right)$. Hence, $\phi=\phi_{1} \times \phi_{2}$ with $\phi_{1} \in \operatorname{Irr}\left(G^{\prime} / N\right)$ and $\phi_{2} \in \operatorname{Irr}(C / N)$. Since $C / N \subseteq \mathbf{Z}(G / N), \phi_{2}$ is $G$-invariant. However, we know that $\phi_{1}$ is not invariant under the diagonal automorphism of $G^{\prime} / N \cong \operatorname{PSL}_{2}(q)$ so that $\phi^{G} \in$ $\operatorname{Irr}(G)$ and thus $\chi=\phi^{G}$, a contradiction. Therefore we conclude that $G / C \cong$ $\operatorname{PSL}_{2}(q)$, and so $G=G^{\prime} \circ C$.

We now show that $C$ is Abelian. Suppose by contradiction that $C$ is nonAbelian and let $\nu \in \operatorname{Irr}(N)$ be a non-trivial character of $N$. Let $\lambda \in \operatorname{Irr}(C)$ with $\lambda(1)>1$. Then $\lambda \in \operatorname{Irr}(C \mid \nu)$ as $C / N$ is Abelian. By Lemma 2.1, $G$ has an irreducible character of degree $(q+1) \lambda(1)$ as $G^{\prime} \cong \mathrm{SL}_{2}(q)$ has a faithful irreducible character of degree $q+1$. However, this is impossible as $q+1$ is the largest character degree of $G$. Therefore, $C$ must be Abelian and the proof is complete.

\section{Step 1 for $\mathrm{SL}_{3}(q)$}

The rest of the paper is devoted to the proof of Theorem 1.3 for $H=\mathrm{SL}_{3}(q)$. Indeed, if $q \not \equiv 1(\bmod 3)$ then $\operatorname{SL}_{3}(q)=\operatorname{PSL}_{3}(q)$, and Theorem 4.1 is exactly Huppert's conjecture for $\operatorname{PSL}_{3}(q)$, which was already solved in [29]. Therefore, to complete the proof of Theorem 1.3 for $\mathrm{SL}_{3}(q)$, it suffices to prove the following.

THEOREM 4.1. Let $G$ be a finite group and $q=p^{f} \geq 4$ be a power of a prime $p$. Suppose that $q \equiv 1(\bmod 3)$. Then $\operatorname{cd}(G)=\operatorname{cd}\left(\mathrm{SL}_{3}(q)\right)$ if and only if $G \cong \mathrm{SL}_{3}(q) \circ A$, where $A$ is Abelian.

The irreducible character degrees of $\mathrm{SL}_{3}(q)$ are available in [7]. For a prime power $7 \leq q \equiv 1(\bmod 3)$, we have

$$
\begin{aligned}
\operatorname{cd}\left(\mathrm{SL}_{3}(q)\right)= & \left\{1, q^{3}, q(q+1),(q-1)^{2}(q+1), q\left(q^{2}+q+1\right),\right. \\
& (q-1)\left(q^{2}+q+1\right)
\end{aligned}
$$




$$
\begin{aligned}
& q^{2}+q+1,(q+1)\left(q^{2}+q+1\right), \frac{1}{3}(q-1)^{2}(q+1), \\
& \left.\frac{1}{3}(q+1)\left(q^{2}+q+1\right)\right\} .
\end{aligned}
$$

When $q=4$ then $\operatorname{cd}\left(\mathrm{SL}_{3}(4)\right)$ is as above excluding $(q+1)\left(q^{2}+q+1\right)$.

To prove Steps 1 and 2, we need to identify the character degrees of $G$ which are non-trivial powers or prime powers. We first observe that $q(q+1)$ is the minimal non-trivial degree of $\mathrm{SL}_{3}(q)$.

LEMMA 4.2. The number $q^{2}+q+1$ is not of the form $y^{n}$ for $y, n \in \mathbb{Z}$ and $n>1$.

Proof. This is Lemma 3.1 of [29].

LEMMA 4.3. The only non-trivial powers among the degrees of $\mathrm{SL}_{3}(q)$ are $q^{3}$ and possibly $q^{3}-1,(q-1)^{2}(q+1)$ and $(q-1)^{2}(q+1) / 3$.

Proof. By [6, Theorem 1], the product $q(q+1)$ of consecutive integers $q$ and $q+1$ is never a non-trivial power. As $q^{2}+q+1$ is not a non-trivial power by Lemma 4.2 and $\operatorname{gcd}\left(q, q^{2}+q+1\right)=\operatorname{gcd}\left(q^{2}+q+1, q+1\right)=1$, we have that $q^{2}+q+1, q\left(q^{2}+q+1\right)$ and $(q+1)\left(q^{2}+q+1\right)$ are not non-trivial powers.

We are left to show that $(q+1)\left(q^{2}+q+1\right) / 3$ is not a non-trivial power. Suppose by contradiction that $(q+1)\left(q^{2}+q+1\right) / 3=y^{n}$ for some integers $n \geq 2$ and $y \geq 2$. Since $3 \mid q^{2}+q+1$ and $\operatorname{gcd}\left(q+1,\left(q^{2}+q+1\right) / 3\right)=1$, we deduce that $q^{2}+q+1=3 a^{n}$ and $q+1=b^{n}$ for some integers $a, b \geq 2$ with $y=a b$. By [17], we obtain that $n=2$. Since $b^{2}$ is congruent to 0 or 1 modulo 3 , we deduce that $q=b^{2}-1$ is congruent to -1 or 0 modulo 3 , contradicting our assumption that $q \equiv 1(\bmod 3)$.

Let $\chi \in \operatorname{Irr}(G)$. Then $\chi$ is said to be isolated in $G$ if $\chi(1)$ is divisible by no proper non-trivial character degree of $G$, and no proper multiple of $\chi(1)$ is a character degree of $G$.

LEMMA 4.4. For $q>2$, the character degrees $q^{3}, q(q+1), q\left(q^{2}+q+1\right)$ and $(q-1)^{2}(q+1)$ are maximal with respect to divisibility among the degrees of $\mathrm{SL}_{3}(q)$. Moreover, $q^{3}$ and $q(q+1)$ are isolated degrees of $\mathrm{SL}_{3}(q)$.

Proof. The degrees $q^{3},(q-1)^{2}(q+1), q(q+1)$ are maximal with respect to divisibility among the degrees of $\operatorname{SL}_{3}(q)$ since $\operatorname{gcd}\left(q,(q-1)^{2}(q+1)\right)=$ $1, \operatorname{gcd}\left(q^{3},(q-1)^{2}(q+1)\right)=1, \operatorname{gcd}\left(q, q^{2}+q+1\right)=\operatorname{gcd}\left(q^{3}, q^{2}+q+1\right)=1$, $\operatorname{gcd}\left(q+1, q^{2}+q+1\right)=1, \operatorname{gcd}(q, q+1)=\operatorname{gcd}\left(q^{3}, q+1\right)=1$ and $\operatorname{gcd}\left(q^{2}+q+\right.$ $\left.1,(q-1)^{2}(q+1)\right)=1$. Furthermore, we observe that both $q^{3}$ and $q(q+1)$ have no non-trivial proper divisor in $\operatorname{cd}\left(\mathrm{SL}_{3}(q)\right)$.

We are now ready to establish Step 1.

Proposition 4.5. Under the hypotheses of Theorem 4.1, $G^{\prime}=G^{\prime \prime}$. 
Proof. By way of contradiction, suppose $G^{\prime} \neq G^{\prime \prime}$. Then there exists a normal subgroup $N$ of $G$ such that $G / N$ is a solvable group and is minimal with respect to being non-Abelian. By Lemma 2.2, $G / N$ is a Frobenius group or an $r$-group for some prime $r$.

(1) $G / N$ is an $r$-group for some prime $r$. As $G / N$ is non-Abelian, it has an irreducible character $\eta$ of degree $r^{b}>1$. We now consider the cases $r=p$ and $r \neq p$ separately.

(1a) $r=p$. We observe that $q^{3}$ is the only non-trivial $p$-power character degree of $G$, and hence $r^{b}=q^{3} \in \operatorname{cd}(G / N)$. Let $\chi \in \operatorname{Irr}(G)$ with $\chi(1)=q^{2}+q+1$. Then $r \nmid \chi(1)$ and by Lemma 2.3(a), $\chi_{N} \in \operatorname{Irr}(N)$. By Lemma 2.3(b), we then have $\eta \chi \in \operatorname{Irr}(G)$, whence $\eta(1) \chi(1)=q^{3}\left(q^{2}+q+1\right) \in \operatorname{cd}(G)$, a contradiction.

(1b) $r \neq p$. Let $\chi \in \operatorname{Irr}(G)$ with $\chi(1)=q^{3}$. Then $\operatorname{gcd}(r, \chi(1))=1$, so that $\operatorname{gcd}(|G / N|, \chi(1))=1$, and thus $\chi_{N} \in \operatorname{Irr}(N)$. We then have $\eta \chi \in \operatorname{Irr}(G)$, and hence $q^{3}<r^{b} q^{3} \in \operatorname{cd}(G)$, which is impossible.

(2) $G / N$ is a Frobenius group with elementary Abelian Frobenius kernel $F / N$, where $|F: N|=r^{c}$ for some prime $r$. In addition, $l=|G: F| \in \operatorname{cd}(G)$.

(2a) $r \neq p$. Let $\chi \in \operatorname{Irr}(G)$ with $\chi(1)=q^{3}$. As $r \nmid \chi(1)$ and no proper multiples and no proper divisors of this degree are in $\operatorname{cd}(G)$, we deduce from Lemma 2.2(b)(ii) that $l=q^{3}$. Let $\varphi \in \operatorname{Irr}(G)$ with $\varphi(1)=q(q+1)$ and let $\psi \in \operatorname{Irr}(F)$ be an irreducible constituent of $\varphi_{F}$. Then $(\varphi(1) / \psi(1))|| G: F \mid=$ $q^{3}$, which implies that $(q+1) \mid \psi(1)$. Since $r \neq p$, by Lemma 2.2(b) we have $r^{c} \mid \psi(1)_{p^{\prime}}^{2}$. As $q \geq 4$ and $|G: F| \mid\left(r^{c}-1\right)$, we have

$$
l=q^{3} \leq r^{c}-1 \leq \psi(1)_{p^{\prime}}^{2}-1<(q+1)^{2}=q^{2}+2 q+1<q^{3},
$$

which is a contradiction.

(2b) $r=p$. We have $|G: F|=l \in \operatorname{cd}(G)$ and $l \mid\left(r^{c}-1\right)=\left(p^{c}-1\right)$. Therefore, the character degree $q(q+1)$ does not divide $l$, and it follows from Lemma 2.2(b)(i) that $r^{c} \mid q^{2}(q+1)^{2}$. Hence, $r^{c} \mid q^{2}$. We now have $l \mid\left(r^{c}-1\right) \leq q^{2}-1$ and hence $l \leq q^{2}-1$. This is impossible since the smallest non-trivial character degree of $G$ is $q(q+1)$.

\section{Step 2 for $\mathrm{SL}_{3}(q)$}

Suppose $G^{\prime} / M$ is a chief factor of $G$. By Step 1 , we have $G^{\prime} / M \cong S^{k}$ for some integer $k \geq 1$ and some non-Abelian simple group $S$. We will show in this section that

$$
G^{\prime} / M \cong S^{k} \cong \operatorname{PSL}_{3}(q)
$$

By the classification of the finite non-Abelian simple groups, $S$ is one of the 26 sporadic simple groups, the Tits group, an alternating groups $\mathrm{A}_{n}$ for $n \geq 7$, or a non-Abelian simple group of Lie type. (Note that $\mathrm{A}_{5} \cong \mathrm{PSL}_{2}(5) \cong$ $\mathrm{PSL}_{2}(4)$ and $\mathrm{A}_{6} \cong \mathrm{PSL}_{2}(9)$, so $\mathrm{A}_{5}$ can $\mathrm{A}_{6}$ can be considered as simple groups of Lie type.) We must show that $k=1$ and $S \cong \operatorname{PSL}_{3}(q)$. 


\subsection{Eliminating the alternating, sporadic, and the Tits groups.}

Proposition 5.1. If $S$ is an alternating group $\mathrm{A}_{n}$ with $n \geq 7$, a sporadic simple group, or the Tits group, then $k=1$.

Proof. By way of contradiction, assume that $k>1$. By Lemma 2.6, $S$ has nonlinear irreducible characters $\psi_{i}, 1 \leq i \leq 5$, of distinct degrees which extend to $\operatorname{Aut}(S)$. By Lemma 2.5, we have that $\psi_{i}^{k}, 1 \leq i \leq 5$, extend to $G$. Thus $G$ has at least 5 non-linear irreducible characters whose degrees are distinct nontrivial powers, which contradicts Lemma 4.3. Therefore, $k=1$ as required.

Proposition 5.2. If $k=1$, then $S$ is not an alternating group $\mathrm{A}_{n}$ with $n \geq 7$.

Proof. As $n \geq 7, S \cong \mathrm{A}_{n}$ has irreducible characters $\theta_{i}, 1 \leq i \leq 3$, which extend to $\operatorname{Aut}(S) \cong \mathrm{S}_{n}$ of degree $n-1, n(n-3) / 2$ and $n(n-3) / 2+1=(n-$ 1) $(n-2) / 2$, respectively. So, $n-1, n(n-3) / 2$ and $(n-1)(n-2) / 2$ are character degrees of $G$, where the last two are consecutive integers. Since $n(n-3) / 2>n-1>1$ and $q(q+1)$ is the non-trivial minimal degree of $G$, we deduce that $n(n-3) / 2=q^{3}-1$ and $q^{3}=(n-1)(n-2) / 2$. (Note that $\left(q(q+1), q^{2}+q+1\right)$ and $\left(q^{3}-1, q^{3}\right)$ are the only pairs of consecutive integers among character degrees of $G$.) Since $n \geq 7$ and $\operatorname{gcd}(n-1, n-2)=1$, we see that the latter equation cannot hold.

Proposition 5.3. If $k=1$, then $S$ is neither a sporadic simple group nor the Tits group.

Proof. For each possibility of $S$, we can find some irreducible characters of $S$ that are extendible to $\operatorname{Aut}(S)$, and then show that the degrees of these characters cannot be all in $\operatorname{cd}(G)=\mathrm{cd}\left(\mathrm{SL}_{3}(q)\right)$. This is straightforward and we skip the details.

5.2. Eliminating the simple groups of Lie type. If $S$ is a simple group of Lie type and $\mathrm{St}_{S}$ is the Steinberg character of $S$, then $\mathrm{St}_{S}(1)$ is a power of the characteristic of the defining field of $S$. By Lemma 2.6, $\mathrm{St}_{S}$ extends to $\operatorname{Aut}(S)$. By Lemma 2.5, it follows that $\operatorname{St}_{S}(1)^{k}$ is a character degree of $G$. As the only prime power among character degrees of $G$ is $q^{3}$, we deduce that $\operatorname{St}_{S}(1)^{k}=q^{3}$. Hence, the defining characteristic of $S$ must be the same as the only prime divisor of $q^{3}$, which is $p$.

We write $S=S\left(q_{1}\right)$ to indicate that $S$ is defined over the field of $q_{1}$ elements. A mixed degree of $S$ is a character degree of $S$ which is divisible by $q_{1}$ but not a power of $q_{1}$. We rely upon the following result on mixed degrees of simple groups of Lie type.

Lemma 5.4 ([26, Lemma 5.6]). If $S$ is a simple group of Lie type and $S \nsubseteq \operatorname{PSL}_{2}\left(q_{1}\right)$, then $S$ possesses an irreducible character of mixed degree. 
Proposition 5.5. If $S$ is a simple group of Lie type and $S \nsupseteq \operatorname{PSL}_{2}\left(q_{1}\right)$, then $k=1$.

Proof. Since $S \nsubseteq \mathrm{PSL}_{2}\left(q_{1}\right)$, by Lemma $5.4, S$ possesses an irreducible character of mixed degree, say $\psi$. Recall that $G^{\prime} / M \cong S^{k}$. Hence, there is an irreducible character of $G^{\prime} / M$ found by multiplying $k-1$ copies of $\mathrm{St}_{S}$ with $\psi$. Then $\left(\operatorname{St}_{S}^{k-1} \psi\right)(1)$ is a mixed degree of $G^{\prime} / M$. As every character degree of $G^{\prime} / M$ divides a degree of $G$, it follows that the degree of this irreducible character divides one of the mixed degrees of $G$. The highest power of $q$ in any mixed degree of $G$ is $q$. Assume that $\operatorname{St}_{S}(1)=q_{1}^{j}$. Then, as $\operatorname{St}_{S}(1)^{k}=q^{3}$, $q_{1}^{j k}=q^{3}$ and hence $q=q_{1}^{j k / 3}$. The power of $q_{1}$ in $\left(\mathrm{St}_{S}^{k-1} \psi\right)(1)$ is at least $q_{1}^{j(k-1)}$. We now have $j(k-1) \leq j k / 3$, which reduces to $2 k \leq 3$. This means that $k=1$, as claimed.

Proposition 5.6. The simple group $S$ is not $\mathrm{PSL}_{2}\left(q_{1}\right)$ for any $k \geq 1$.

Proof. Arguing as in Proposition 5.5, we can show that $k \leq 2$. First, assume that $k=1$. As the degree of the Steinberg character of $\operatorname{PSL}_{2}\left(q_{1}\right)$ is $q_{1}$, we must have $q_{1}=q^{3}$. It follows that $q_{1}+1=q^{3}+1$ is a character of $S$ as well as $G^{\prime}$, and hence $q^{3}+1$ divides a character degree of $G$, which is impossible. Now consider $k=2$. Then $q_{1}^{2}=q^{3}$. Thus $\left(\sqrt{q^{3}}+1\right)^{2}$ is a character degree of $G^{\prime} / M$. This is again impossible since $\left(\sqrt{q^{3}}+1\right)^{2}$ does not divide any character degree of $G$.

Proposition 5.7. $S$ is not a simple group of exceptional Lie type.

Proof. We will examine each family of simple groups of exceptional Lie type.

(1) $S \cong{ }^{2} \mathrm{~B}_{2}\left(q_{1}^{2}\right)$ where $q_{1}^{2}=2^{2 m+1}$ with $m \geq 1$. Recall that

$$
\operatorname{cd}\left({ }^{2} \mathrm{~B}_{2}\left(q_{1}^{2}\right)\right)=\left\{1, q_{1}^{4}, q_{1}^{4}+1,\left(q_{1}^{2}-1\right) a,\left(q_{1}^{2}-1\right) b,\left(q_{1}^{2}-1\right) u\right\},
$$

where $q_{1}^{2}=2^{2 m+1} \geq 8, u=\frac{1}{\sqrt{2}} q_{1}, a=q_{1}^{2}+\frac{1}{\sqrt{2}} q_{1}+1$, and $b=q_{1}^{2}-\frac{1}{\sqrt{2}} q_{1}+1$. The largest character degree of ${ }^{2} \mathrm{~B}_{2}\left(q_{1}^{2}\right)$ is $\left(q_{1}^{2}-1\right) a=\left(q_{1}^{2}-1\right)\left(q_{1}^{2}+\frac{1}{\sqrt{2}} q_{1}+1\right)$. As the Steinberg character of $S$ has a degree $q_{1}^{4}$, we have $q_{1}^{4}=q^{3}$, and so $q_{1}^{4 / 3}=q$.

By [14], the unipotent character of degree $\left(q_{1}^{2}-1\right) u$ of $S$ is extendible to $\operatorname{Aut}(S)$. Hence $u\left(q_{1}^{2}-1\right) \in \operatorname{cd}(G)$. Since $q=q_{1}^{4 / 3}, q$ is even and so $u\left(q_{1}^{2}-1\right)$ is an even degree of $G$ but not a 2 -power, so $u\left(q_{1}^{2}-1\right)=q(q+1)$ or $q\left(q^{2}+q+1\right)$. Comparing the even parts, we deduce that $2^{m}=u=q=2^{(4 m+2) / 3}$, which is absurd.

(2) $S \cong \mathrm{G}_{2}\left(q_{1}\right)$ with $q_{1}>2$. Following the notation for unipotent characters in [4, Section 13.8], the unipotent character $\phi_{2,2}$ of $S$ extends to $\operatorname{Aut}(S)$ and thus $\phi_{2,2}(1)=q_{1}\left(q_{1}+1\right)\left(q_{1}^{3}+1\right) / 2 \in \operatorname{cd}(G)$. By our assumption on $q_{1}$, $\phi_{2,2}(1)$ is divisible by $p$ but not a power of $p$ since $q_{1}^{2}=q$ and $q_{1}^{3}+1$ is prime 
TABLE 1. Some characters of the simple groups of Lie type

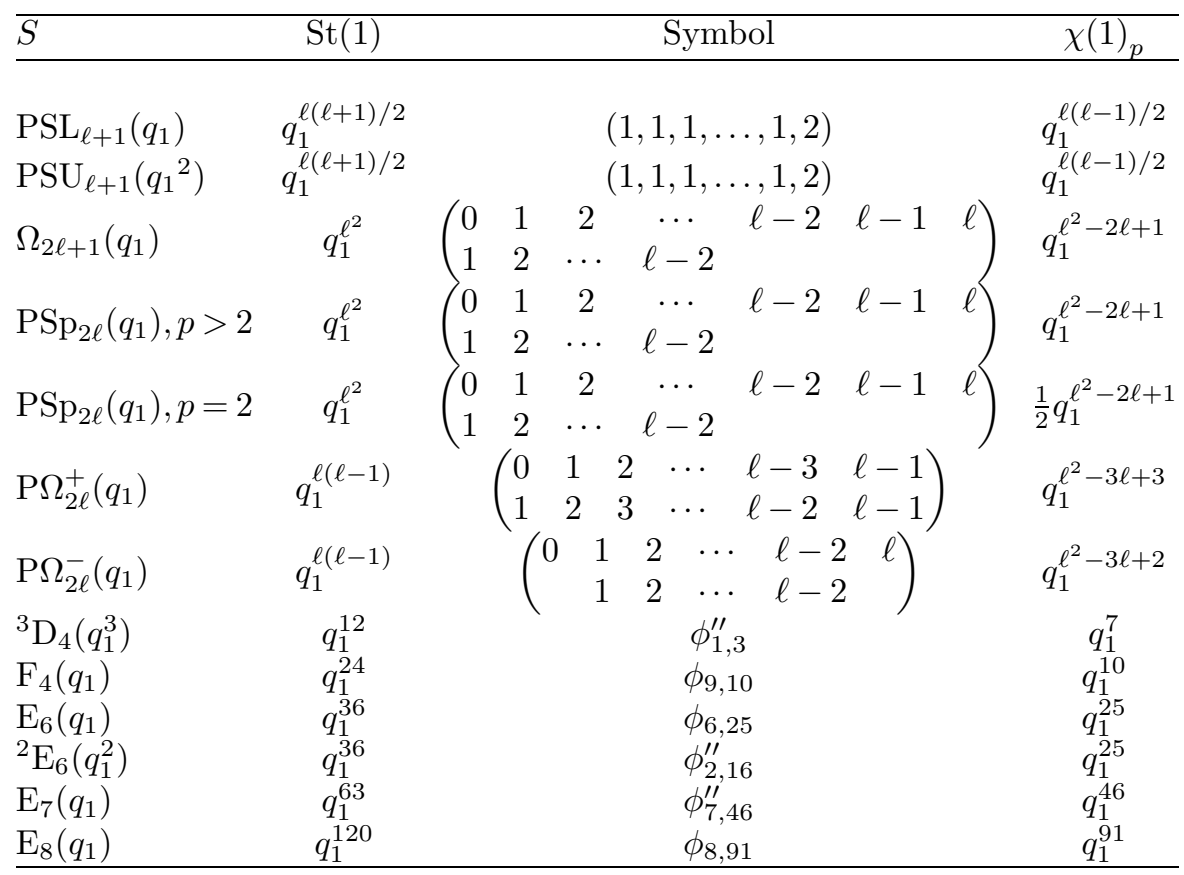

to $p$. So, $\phi_{2,2}(1)=q(q+1)$ or $q\left(q^{2}+q+1\right)$. Now if $p$ is odd, then by comparing the $p$-part, we have $q_{1}=q$ which is impossible as $q=q_{1}^{2}$. If $p$ is even, then $q_{1} / 2=q=q_{1}^{2}$, a contradiction again.

(3) $S \cong{ }^{2} \mathrm{G}_{2}\left(q_{1}^{2}\right), q_{1}^{2}=3^{2 m+1}, m \geq 1$. Consider the unipotent character of $S$ of degree $q_{1}\left(q_{1}^{2}-1\right)\left(q_{1}^{2}+1\right) / \sqrt{3}$. This is a mixed degree, so it must divide either $q(q+1)$ or $q\left(q^{2}+q+1\right)$. This character degree is even as $q_{1}$ is a power of 3. Thus, it must divide $q(q+1)$ since $q\left(q^{2}+q+1\right)$ is odd. The Steinberg character of $S$ has a degree $q_{1}^{6}$, so $q=q_{1}^{2}$. Hence, we have $\left(q_{1}^{2}-1\right)\left(q_{1}^{2}+1\right)$ divides $q_{1}^{2}+1$, a contradiction.

(4) For the remaining simple groups of exceptional Lie type, we argue as follows. Let $S=S\left(q_{1}\right)$ be a simple group of exceptional Lie type defined over a field of $q_{1}$ elements. Now, suppose that the Steinberg character of $S$ has degree $q_{1}^{j}$. By Lemma 2.5, $q^{3}=q_{1}^{j}$, and thus $q=q_{1}^{j / 3}$. For each of the remaining possibilities for $S$, there is a mixed character degree of $S$ whose power on $q_{1}$ is greater than $q_{1}^{j / 3}>q$, as shown in Table 1 , and this is a contradiction. In this table we follow the notation for unipotent characters in [4, Section 13.8]. 
We have already shown that $k=1$. So Step 2 is complete once we have the following.

Proposition 5.8. $S \cong \operatorname{PSL}_{3}(q)$.

Proof. By the previous propositions, we now may assume that $S=S\left(q_{1}\right)$ is a simple classical group defined over a field of $q_{1}$ elements. The Steinberg character of $S$ has degree $q_{1}^{j}$ for some $j$. By Lemma 2.5, $q^{3}=q_{1}^{j}$ and hence $q=q_{1}^{j / 3}$.

(1) $S \cong \mathrm{PSL}_{l+1}\left(q_{1}\right)$ or $S \cong \operatorname{PSU}_{l+1}\left(q_{1}^{2}\right)$ with $l \geq 1$.

Since $k=1$, we must have $l(l-1) / 2 \leq l(l+1) / 6$, which implies that $l \leq 2$. Since the cases $S \cong \operatorname{PSU}_{2}\left(q_{1}^{2}\right)$ and $S \cong \operatorname{PSL}_{2}\left(q_{1}\right)$ were already handled in Proposition 5.6, it remains to assume that $l=2$. It then follows that the Steinberg character of $S$ has degree $q_{1}^{3}$, and therefore by Lemma $2.5, q_{1}^{3} \in$ $\operatorname{cd}(G)$. This forces $q_{1}=q$ so that $S \cong \operatorname{PSL}_{3}(q)$ or $\operatorname{PSU}_{3}\left(q^{2}\right)$.

If $S \cong \operatorname{PSU}_{3}\left(q^{2}\right)$, then $S$ has a mixed degree, namely $q(q-1)$, but this degree does not divide any degree of $G$, a contradiction. We conclude that $S \cong \mathrm{PSL}_{3}(q)$, as claimed.

(2) $S \cong \mathrm{P} \Omega_{2 l}^{ \pm}\left(q_{1}\right)$ with $l \geq 4$.

As shown in Table 1 , we have $l^{2}-3 l+2 \leq l(l-1) / 3$, which is impossible.

(3) $S \cong \Omega_{2 l+1}\left(q_{1}\right)$ or $S \cong \operatorname{PSp}_{2 l}\left(q_{1}\right)$ with $l \geq 2$.

As shown in Table 1 , we must have $l^{2}-2 l+1 \leq l^{2} / 3$, which is not satisfied for $l \geq 3$. If $q_{1}$ is even, then the exponent on $q_{1}$ in the degree is at least $l^{2}-2 l+1-(l-1)$. But $l^{2}-2 l+1-(l-1) \leq l^{2} / 3$ is not satisfied for $l \geq 4$. Suppose that $l=2$. For $q_{1}=3$, the degree of the Steinberg character is $3^{4}$, which cannot possibly extend to $q^{3}$. Assume $q_{1} \neq 3$. Note that $S$ possesses unipotent characters of degree $q_{1}\left(q_{1}^{2}+1\right) / 2$ and $q_{1}\left(q_{1}-1\right)^{2} / 2$. The degree of the Steinberg character of $S$ is $q_{1}^{4}$. Thus, we must have that $q=q_{1}^{4 / 3}$. But these degrees cannot divide $q_{1}^{4 / 3}\left(q_{1}^{4 / 3}+1\right)$ and so they must divide $q\left(q^{2}+q+1\right)=$ $q_{1}^{4 / 3}\left(q_{1}^{8 / 3}+q_{1}^{4 / 3}+1\right)$. Both these degrees must divide $q\left(q^{2}+q+1\right)$. Hence, $q^{2}+q+1$ has factors $q_{1}-1$ and $q_{1}^{2}+1$. This implies $q^{2}+q+1$ has a divisor congruent -1 modulo 3, contradicting [29, Lemma 2.1].

Now suppose that $l=3$. From [4, Section 13.8], we see that $S$ has a unipotent character $\chi^{\alpha}$ of degree

$$
\chi^{\alpha}(1)=\frac{1}{2} q_{1}^{4}\left(q_{1}+1\right)\left(q_{1}^{2}-q_{1}+1\right)
$$

corresponding to the symbol

$$
\alpha=\left(\begin{array}{lllll}
1 & & 2 & & 3 \\
& 0 & & 1 &
\end{array}\right) .
$$

When $l=3$, the degree of the Steinberg character of $S$ is $q_{1}^{9}$, so we have $q=q_{1}^{3}$. Hence, $\chi^{\alpha}(1)$ divides no degree of $G$. The proof is complete. 


\section{Step 3 for $\mathrm{SL}_{3}(q)$}

In this section, we will prove that $G^{\prime}$ is isomorphic to a perfect central cover of $\operatorname{PSL}_{3}(q)$, and then deduce that $G^{\prime}$ must be either $\operatorname{SL}_{3}(q)$ or $\operatorname{PSL}_{3}(q)$. We begin with a known lemma on maximal subgroups of $\mathrm{SL}_{2}(q)$.

Lemma 6.1. ([27, Lemma 6]) Assume that $q \geq 3$ is a prime power. If $M$ is a maximal subgroup of $\mathrm{SL}_{2}(q)$ whose index divides $q \pm 1$ or $q$, then one of the following cases holds.

(a) If $q \geq 13$ is odd or $q \geq 4$ is even, then $M$ is the Borel subgroup of index $q+1$. Moreover, $q+1$ is the smallest index of a maximal subgroup of $\mathrm{SL}_{2}(q)$.

(b) If $q \in\{3,5,7,11\}$, then either $M$ is the Borel subgroup of index $q+1$ or $M$ is a non-Abelian subgroup of index $q$.

(c) If $q=9$, then $M$ is the Borel subgroup of index 10 and 6 is the smallest index of a maximal subgroup of $\mathrm{SL}_{2}(9)$.

LEMMA 6.2. Let $q \geq 4$ be a prime power and $q \equiv 1(\bmod 3)$. Let $K \unlhd L$ be such that $L / K \cong \mathrm{SL}_{2}(q)$ and let $\delta \in \operatorname{Irr}(K)$. If $\chi(1)$ divides $q$ or $q \pm 1$ for every $\chi \in \operatorname{Irr}(L \mid \delta)$, then $\delta$ is L-invariant.

Proof. Suppose by contradiction that $\delta$ is not $L$-invariant and let $V:=$ $I_{L}(\delta)$. Write

$$
\delta^{V}=\sum_{i} \eta_{i} \quad \text { where } \eta_{i} \in \operatorname{Irr}(V \mid \delta) .
$$

It follows that, for each $i, \eta_{i}^{L} \in \operatorname{Irr}(L \mid \delta)$ and $\eta_{i}^{L}(1)=|L: V| \eta_{i}(1)$ divides $q$ or $q \pm 1$. Let $M$ be a subgroup of $L$ such that $V \leq M$ and $M / K$ is maximal in $L / K$. From Lemma 6.1, since $|L: M|$ divides $q \pm 1$ or $q$, we deduce that $|L: M|=q$ and $q \in\{7,11\}$ or $|L: M|=q+1$ and $M / K$ is the Borel subgroup of $L / K$.

First, we consider the case $q=7$ or 11 and $|L: M|=q$. Then $M / K$ is a non-Abelian subgroup of $L / K$ of index $q$. It follows that

$$
\eta_{i}^{L}(1)=|L: M| \cdot|M: V| \eta_{i}(1)=q \cdot|M: V| \eta_{i}(1)
$$

divides $q$, which forces $M=V$ and $\eta_{i}(1)=1$ for every $i$. Hence, $V / K=$ $M / K$ is Abelian as $\delta$ extends to $V$ and all irreducible characters of $V / K$ are linear. However, this leads to a contradiction as $M / K$ is non-Abelian by Lemma 6.1(b).

Now assume $M / K$ is isomorphic to the Borel subgroup of $L / K$ and $\mid L$ : $M \mid=q+1$. Since $\eta_{i}^{L}(1)$ divides $q$ or $q \pm 1$, we have

$$
\eta_{i}^{L}(1)=|L: M| \cdot|M: V| \eta_{i}(1)=(q+1) \cdot|M: V| \eta_{i}(1)
$$

must divide $q+1$ and we deduce that $|M: V| \eta_{i}(1)=1$. This implies that $M=V$ and $\eta_{i}(1)=1$ for every $i, M / K=V / K$ is isomorphic to the Borel subgroup of $\mathrm{SL}_{2}(q)$ of index $q+1$ and that all constituents of $\delta^{V}$ are linear. 
TABLE 2. Maximal subgroups of $\mathrm{PSL}_{2}(q)$ (see [12], [24])

\begin{tabular}{lcc}
\hline Subgroup & Condition & Index \\
\hline $\mathrm{D}_{(q-1)}$ & $q \geq 13$, odd & $\frac{1}{2} q \Phi_{2}$ \\
$\mathrm{D}_{2(q-1)}$ & $q$ even & $\frac{1}{2} q \Phi_{2}$ \\
$\mathrm{D}_{(q+1)}$ & $q \neq 7,9$, odd & $\frac{1}{2} q \Phi_{1}$ \\
$\mathrm{D}_{2(q+1)}$ & $\frac{1}{2} q \Phi_{1}$ \\
Borel subgroup & $q$ even & $\Phi_{2}$ \\
$\mathrm{PSL}_{2}\left(q_{0}\right)(2, \alpha)$ & $q=q_{0}^{\alpha}$ & \\
$\mathrm{S}_{4}$ & $q=p \equiv \pm 1 \bmod 8$ & \\
& $q=p^{2}, 3<p \equiv \pm 3 \bmod 10$ & \\
$\mathrm{~A}_{4}$ & $q=p \equiv \pm 3 \bmod 8, q>3$ \\
$\mathrm{~A}_{5}$ & $q=p \equiv \pm 1 \bmod 10$ & \\
& $q=p^{2}, p \equiv \pm 3 \bmod 10$ & \\
\hline
\end{tabular}

By Lemma 2.3(b), we deduce that the Borel subgroup $M / K$ is Abelian. This contradiction completes the proof.

Lemma 6.3. Let $q \geq 7$ be a prime power such that $q \equiv 1(\bmod 3)$. Let $X$ be a perfect group and $M \triangleleft X$ such that $X / M$ is a cover of $\operatorname{PSL}_{3}(q)$ and every character degree of $X$ divides a character degree of $\mathrm{SL}_{3}(q)$. Then every linear character of $M$ is stable under $X$.

Proof. Let $\vartheta \in \operatorname{Irr}(M)$ with $\vartheta(1)=1$. Assume to the contrary that $\vartheta$ is not stable under $X$, we have $I:=I_{X}(\vartheta) \lesseqgtr X$. Hence, $I$ is contained in a maximal subgroup, say $P$, of $X$. Let

$$
\vartheta^{I}=\sum_{i} \varphi_{i} \quad \text { for } \varphi_{i} \in \operatorname{Irr}(I)
$$

By Lemma $2.4, \varphi_{i}(1)|X: I|$ is a character degree of $X$ and hence it divides some character degree of $\mathrm{SL}_{3}(q)$.

Thus, we will need to find indices of maximal subgroups of $\operatorname{PSL}_{3}(q)$ which divide some character degrees of $\mathrm{SL}_{3}(q)$. From the list of maximal subgroups of $\operatorname{PSL}_{2}(q)$ and $\operatorname{PSL}_{3}(q)$ in [12], [24], we produce Tables 2 and 3. In these tables, $\left[q^{2}\right]$ denotes an unspecified group of order $q^{2}, A: B$ denotes a split extension, $A \circ B$ denotes a central product, and $A \cdot B$ denotes a non-split extension.

We first notice that $X / M \cong \mathrm{SL}_{3}(q)$ or $\mathrm{PSL}_{3}(q)$ since $7 \leq q \equiv 1(\bmod 3)$ and the Schur multiplier of $\mathrm{PSL}_{3}(q)$ is cyclic of order 3. Moreover, the sets of indices of maximal subgroups of $\mathrm{SL}_{3}(q)$ and $\mathrm{PSL}_{3}(q)$ are the same.

By inspecting Table 3 , the only possibility for $P / M$ is $\left[q^{2}\right]: \mathrm{GL}_{2}(q)$ or its image in $\mathrm{PSL}_{3}(q)$ according to whether $X / M \cong \mathrm{SL}_{3}(q)$ or $\mathrm{PSL}_{3}(q)$. Then $P / M \cong\left[q^{2}\right]: \mathrm{GL}_{2}(q)=\left[q^{2}\right]: \mathrm{SL}_{2}(q)(q-1)$ or $\left[q^{2}\right]: \mathrm{SL}_{2}(q)(q-1) / 3$. 
TABLE 3. Maximal subgroups of $\mathrm{PSL}_{3}(q)$ (see [7], [12], [24])

\begin{tabular}{lc}
\hline Subgroup & Condition \\
\hline$\wedge\left[q^{2}\right]: \mathrm{GL}_{2}(q)$ & \\
$\wedge\left(\mathbb{Z}_{q-1}\right)^{2} \cdot \mathrm{S}_{3}$ & $q \geq 5$ \\
$\wedge \mathbb{Z}_{q^{2}+q+1} \cdot 3$ & $q \neq 4$ \\
$\mathrm{PSL}\left(q_{0}\right) \cdot((q-1,3), b)$ & $q=q_{0}^{b}, b$ prime \\
$3^{2} \cdot \mathrm{SL}_{2}(3)$ & $q=p \equiv 1 \bmod 9$ \\
$3^{2} \cdot \mathrm{Q}_{8}$ & $q=p \equiv 4,7 \bmod 9$ \\
$\mathrm{SO}_{3}(q)$ & $q$ odd \\
$\mathrm{PSU}_{3}\left(q_{0}\right)$ & $q=q_{0}^{2}$ \\
$\mathrm{~A}_{6}$ & $p \equiv 1,2,4,7,8,13 \bmod 15$ \\
$\mathrm{PSL}_{2}(7)$ & $2<q=p \equiv 1,2,4 \bmod 7$ \\
\hline
\end{tabular}

We have that $|X: P|=q^{2}+q+1$ and for every $i,|P: I| \varphi_{i}(1)$ divides $q$ and $q \pm 1$. Let $S \unlhd T$ be normal subgroups of $P$ such that $S / M \cong\left[q^{2}\right]$, and $T / S \cong \mathrm{SL}_{2}(q)$.

Observe that if $\chi \in \operatorname{Irr}(T \mid \vartheta)$, then $\chi(1)$ divides $q$ or $q \pm 1$.

Assume $S \leq I \leq P$. Write

$$
\vartheta^{S}=\sum_{i=1}^{k} \delta_{i} \quad \text { where } \delta_{i} \in \operatorname{Irr}(S \mid \vartheta) .
$$

We now show that $S / \operatorname{Ker} \vartheta$ is Abelian. It suffices to show that $S^{\prime} \leq \operatorname{Ker} \delta_{i}$ for all $i$ since

$$
\bigcap_{i=1}^{k} \operatorname{Ker} \delta_{i}=\operatorname{Ker} \vartheta^{S} \leq \operatorname{Ker}(\vartheta) \leq M .
$$

Suppose by contradiction that $S^{\prime} \not \mathbf{K e r} \delta_{j}$, for some $j$, hence $1=\vartheta(1)<\delta_{j}(1)$ and $p \mid \delta_{j}(1)$. As $\operatorname{Irr}\left(T \mid \delta_{j}\right) \subseteq \operatorname{Irr}(T \mid \vartheta)$, by Lemma 6.2 , we see that $\delta_{j}$ is $T$-invariant.

As $q \equiv 1(\bmod 3)$, we observe that $q \neq 9$ so the Schur multiplier of $T / S \cong$ $\mathrm{SL}_{2}(q)$ is trivial. From [10, Theorem 11.7], $\delta_{j}$ extends to $\delta_{0} \in \operatorname{Irr}(T)$ and hence by Lemma $2.3(\mathrm{~b}), \delta_{0} \eta$ are all the irreducible constituents of $\delta^{T}$ for $\eta \in \operatorname{Irr}(T / S)$. Taking $\eta \in \operatorname{Irr}(T / S)$ with $\eta(1)=q$, we then see that $\delta_{0}(1) \eta(1)=$ $q \delta_{j}(1)$ divides $q$ and $q \pm 1$, which is impossible. Hence, $S / \operatorname{Ker} \vartheta$ is Abelian. By Lemma 6.2, each linear $\delta_{i}$ is $T$-invariant. Therefore,

$$
[T, S] \leq \bigcap_{i=1}^{k} \operatorname{Ker} \delta_{i} \leq \operatorname{Ker} \vartheta^{S} \leq M,
$$

which is a contradiction as $T$ acts non-trivially on $S / M \cong\left[q^{2}\right]$.

Assume $S \not \leq I \leq P$. Since $I \cap S \leq S$, one can find $\delta \in \operatorname{Irr}(S \mid \vartheta)$ with $p \mid \delta(1)$. Since $\operatorname{Irr}(T \mid \delta) \subseteq \operatorname{Irr}(T \mid \vartheta)$, for every $\chi \in \operatorname{Irr}(T \mid \delta), \chi(1)$ divides $q$ or $q \pm 1$ and 
TABLE 4. Maximal subgroups of $\mathrm{PSL}_{3}(4)$

\begin{tabular}{lcr}
\hline Structure & Order & Index \\
\hline $2^{4}: \mathrm{A}_{5}$ & 960 & 21 \\
$\mathrm{~A}_{6}$ & 360 & 56 \\
$\mathrm{PSL}_{2}(7)$ & 168 & 120 \\
$3^{2}: Q_{8}$ & 72 & 280 \\
\hline
\end{tabular}

hence by Lemma $6.2, \delta$ is $T$-invariant. Now since $q \geq 7, q \neq 9$, the Schur multiplier of $T / S \cong \mathrm{SL}_{2}(q)$ is trivial and by [10, Theorem 11.7] $\delta$ extends to $\delta_{0} \in \operatorname{Irr}(T \mid \vartheta)$. Hence, by Lemma 2.3(b), $\delta_{0} \eta$ are all the irreducible constituents of $\delta^{T}$ for $\eta \in \operatorname{Irr}(T / S)$. Taking $\eta \in \operatorname{Irr}(T / S)$ with $\eta(1)=q, \delta_{0}(1) \eta(1)=q \delta(1)$ must divide $q$ or $q \pm 1$, which is impossible.

Lemma 6.4. Let $X$ be a perfect group and $M \triangleleft X$ such that $X / M$ is a cover of $\mathrm{PSL}_{3}(4)$ and every character degree of $X$ divides a degree of $\mathrm{SL}_{3}(4)$. Then every linear character of $M$ is stable under $X$.

Proof. The hypotheses imply that $X / M$ is isomorphic to $\mathrm{PSL}_{3}(4)$ or $\mathrm{SL}_{3}(4)$ since any other cover of $\mathrm{PSL}_{3}(4)$ has a character degree that does not divide a degree of $\mathrm{SL}_{3}(4)$. Let $\zeta \in \operatorname{Irr}(M)$ with $\zeta(1)=1$. By way of contradiction, suppose $\zeta$ is not invariant under $X$. Then the inertia group $I_{X}(\zeta)$ of $\zeta$ is contained in a maximal subgroup of $X$.

We will prove that there is no such maximal subgroup. Let $I:=I_{X}(\zeta) \lesseqgtr X$ and let $P$ be maximal such that $I \leq P \leq X$. If

$$
\zeta^{I}=\sum_{i} \xi_{i} \quad \text { for } \xi_{i} \in \operatorname{Irr}(I)
$$

then, by Lemma 2.4, $\xi_{i}(1)|X: I|$ is a character degree of $X$ and thus divides some degree of $\mathrm{SL}_{3}(4)$. Hence, we need to all maximal subgroups $P / M$ of $X / M$ such that $|X: P|$ divides some character degrees of $\mathrm{SL}_{3}(4)$. Recall that $X / M$ is isomorphic to $\mathrm{PSL}_{3}(4)$ or $\mathrm{SL}_{3}(4)$.

Maximal subgroups of $\mathrm{PSL}_{3}(4)$ are available in Atlas [5] and listed in Table 4 . The corresponding maximal subgroups of $\mathrm{SL}_{3}(4)$ are

$$
3 \times 2^{4}: \mathrm{A}_{5}, 3 \cdot \mathrm{A}_{6}, 3 \times \mathrm{PSL}_{2}(7), 3_{+}^{1+2}: \mathrm{Q}_{8} .
$$

Inspecting the character degrees of $\mathrm{SL}_{3}(4)$, we observe that the index $\mid X$ : $P \mid$ divides some degree $\mathrm{SL}_{3}(4)$ only if $|X: P|=21$.

It follows that $P / M \cong 2^{4}: \mathrm{A}_{5}$ or $3 \times 2^{4}: \mathrm{A}_{5}$ and $|P: I| e_{i}(1)$ divides 4 for every $i$. Using GAP [3], we can check that $P / M$ has no maximal subgroup whose index divides 4 , therefore $P=I$ and that $\xi_{i}(1)$ is a power of 2 for all $i$ 's. By Lemma 2.8, $P / M$ must be solvable which is impossible. Thus, every linear character of $M$ is $X$-invariant as wanted. 
We now complete the proof of Step 3. By combining Steps 1 and 2, we have $G^{\prime} / M \cong \operatorname{PSL}_{3}(q)$. As every character degree of $G^{\prime}$ divides a character degree of $G$, Lemmas 6.3 and 6.4 imply that every linear character of $M$ is stable under $G^{\prime}$. It follows from $\left[9\right.$, Lemma 6] that $\left|M: M^{\prime}\right| \operatorname{divides}\left|\operatorname{Mult}\left(G^{\prime} / M\right)\right|=$ $\left|\operatorname{Mult}\left(\mathrm{PSL}_{3}(q)\right)\right|$. Lemma 2.10 then yields that $G^{\prime} / M^{\prime}$ is isomorphic to a cover of $\operatorname{PSL}_{3}(q)$.

Repeating the above arguments by using Lemmas 6.3, 6.4, 2.10, and [9, Lemma 6], we deduce that $G^{\prime} / M^{(i)}$ is isomorphic to a cover of $\operatorname{PSL}_{3}(q)$ for every $i \geq 0$. Therefore, if $M$ is solvable then $G^{\prime}$ is isomorphic to a cover of $\operatorname{PSL}_{3}(q)$. This immediately implies that $G^{\prime}$ is isomorphic to $\operatorname{PSL}_{3}(q)$ or $\operatorname{SL}_{3}(q)$ if $q \geq 7$. When $q=4$, we have the same conclusion by noticing that any cover of $\mathrm{PSL}_{3}(4)$ different from $\mathrm{PSL}_{3}(4)$ and $\mathrm{SL}_{3}(4)$ has a character degree which does not divide a degree of $\mathrm{SL}_{3}(4)$.

The proof of Step 3 is complete once we can show that $M$ cannot be nonsolvable. Assume so, then there is an integer $i$ such that

$$
M^{(i)}=M^{(i+1)}>1 \text {. }
$$

Let $N \leq M^{(i)}$ be a normal subgroup of $G^{\prime}$ so that $M^{(i)} / N \cong T^{k}$ for some nonAbelian simple group $T$. By [16, Lemma 4.2], $T$ has a non-principal irreducible character $\varphi$ that extends to Aut $(T)$. Now Lemma 2.5 implies that $\varphi^{k}$ extends to $\Phi \in \operatorname{Irr}(G / N)$. Therefore, by Gallagher's theorem, $\Phi \chi \in \operatorname{Irr}\left(G^{\prime} / N\right)$ for every $\chi \in \operatorname{Irr}\left(G^{\prime} / M^{(i)}\right)$. In particular,

$$
\varphi(1)^{k} \chi(1) \in \operatorname{cd}\left(G^{\prime} / N\right) \subseteq \operatorname{cd}\left(G^{\prime}\right) .
$$

However, we have that $G^{\prime} / M^{(i)}$ is isomorphic to a cover of $\operatorname{PSL}_{3}(q)$. Choosing $\chi$ to be the Steinberg character of degree $q^{3}$ of $G^{\prime} / M^{(i)}$, we deduce that $q^{3} \varphi^{k}(1)$ is a character degree of $G^{\prime}$ and therefore it divides a degree of $G$. This is a contradiction by inspecting $\operatorname{cd}\left(\mathrm{SL}_{3}(q)\right)$.

\section{Step 4 for $\mathrm{SL}_{3}(q)$}

In this section, we aim to show that $G=G^{\prime} \circ C$ where $C:=\mathbf{C}_{G}\left(G^{\prime}\right)$ is Abelian. From Step 3, we know that $G^{\prime}$ is isomorphic to either $\operatorname{PSL}_{3}(q)$ or $\mathrm{SL}_{3}(q)$. We deduce that $G / C$ is an almost simple group with socle $\operatorname{PSL}_{3}(q)$. In other words,

$$
\mathrm{PSL}_{3}(q) \unlhd G / C \leq \operatorname{Aut}\left(\mathrm{PSL}_{3}(q)\right) .
$$

As $q \geq 4$ and $q \equiv 1(\bmod 3)$, by [8, Theorem 2.5.12], we have

$$
\operatorname{Out}\left(\operatorname{PSL}_{3}(q)\right)=\langle d\rangle:(\langle\sigma\rangle \times\langle\tau\rangle)
$$

where $\tau$ is a graph automorphism of order $2, \sigma$ is a field automorphism of order $f$ with $q=p^{f}$, and $d$ is a diagonal automorphism of order 3 .

(1) First, we show that $C$ is Abelian. Let $Z:=\mathbf{Z}\left(G^{\prime}\right)$. Then $Z$ is normal in $G$ and $G^{\prime} / Z \times C / Z \unlhd G / Z$. Assume that $C / Z$ is non-Abelian. We then can find $\lambda \in \operatorname{Irr}(C / Z)$ with $\lambda(1)>1$ and let $\mu \in \operatorname{Irr}\left(G^{\prime} / Z\right)$ with $\mu(1)=q^{3}$. 
Then we know that $\mu \times \lambda \in \operatorname{Irr}\left(G^{\prime} / Z \times C / Z\right)$ and so $(\mu \times \lambda)(1)=q^{3} \lambda(1)$ must divide some character degree of $G$, which is impossible as $q^{3} \lambda(1)>q^{3}$. We have shown that $C / Z$ is Abelian.

If $|Z|=1$ then $C=C / Z$ is Abelian as we wanted. So it remains to assume that $|Z|=3$ so that $G^{\prime} \cong \mathrm{SL}_{3}(q)$. By [7], $\mathrm{SL}_{3}(q)$ has a faithful irreducible character of degree $q\left(q^{2}+q+1\right)$. Hence, there exist $1 \neq \nu \in \operatorname{Irr}(Z)$ and $\mu \in \operatorname{Irr}\left(G^{\prime} \mid \nu\right)$ with $\mu(1)=q\left(q^{2}+q+1\right)$. Assume by contrary that $C$ is non-Abelian. Then, as $C / Z$ is non-Abelian, we can find $\lambda \in \operatorname{Irr}(C \mid \nu)$ such that $\lambda(1)>1$. Using Lemma 2.1, we deduce that there exists $\chi \in \operatorname{Irr}\left(G^{\prime} \circ C\right)$ of degree $\mu(1) \lambda(1)=\lambda(1) q\left(q^{2}+q+1\right)$. It follows that $\lambda(1) q\left(q^{2}+q+1\right)$ divides some character degree of $G$, which is impossible as $q\left(q^{2}+q+1\right)$ is maximal with respect to divisibility among the character degrees of $\mathrm{SL}_{3}(q)$. We therefore conclude that $C$ is Abelian.

(2) Next, we claim that every irreducible character of $C$ is $G$-invariant. Assume to the contrary that $\lambda \in \operatorname{Irr}(C)$ is not $G$-invariant. If $Z$ lies in the kernel of $\lambda$, then $\chi=\mathrm{St}_{G^{\prime} / Z} \times \lambda$ is an irreducible character of $G^{\prime} C / Z$ and is not $G$-invariant, and it follows that $G$ has a character degree which is a proper multiple of $q^{3}$, a contradiction. So $Z$ does not lie in $\operatorname{Ker}(\lambda)$. In particular, $Z$ is nontrivial and $G^{\prime} \cong \mathrm{SL}_{3}(q)$. Suppose that $\lambda \in \operatorname{Irr}(C \mid \nu)$ where $\nu \in \operatorname{Irr}(Z)$ is nontrivial. We know that $G^{\prime} \cong \mathrm{SL}_{3}(q)$ has a faithful irreducible character $\mu$ of degree $(q-1)^{2}(q+1)$ and is $G$-invariant. (This follows from the fact that no proper multiple of $(q-1)^{2}(q+1)$ is a degree of $\mathrm{SL}_{3}(q)$.) So, $\chi=\mu \lambda$ is an irreducible character of $G^{\prime} C$ which is not $G$-invariant, and thus $G$ has a character degree which is a proper multiple of $(q-1)^{2}(q+1)$ which is impossible.

(3) We finally show that $G=G^{\prime} \circ C$. Assume not, then $G / C$ induces a non-trivial outer automorphism on $G^{\prime}$, say $\alpha$. We shall show that $\operatorname{cd}(G / C) \nsubseteq$ $\operatorname{cd}\left(\mathrm{SL}_{3}(q)\right)$, and this violates the hypothesis.

(3i) Assume $\alpha=d^{a} \tau$ with $0 \leq a<3$. By a result of Zsigmondy [31], we can find $\omega \in \overline{\mathbb{F}_{p}}$ of order which is a primitive prime divisor of $p^{3 f}-1=q^{3}-1$; that is a prime divisor of $p^{3 f}-1$ that does not divide $\prod_{i=1}^{3 f-1}\left(p^{i}-1\right)$. We then consider a semisimple element $s \in \mathrm{SL}_{3}(q)$ with eigenvalues $\omega, \omega^{q}$ and $\omega^{q^{2}}$. The image of $s$ under the canonical projection $\mathrm{GL}_{3}(q) \rightarrow \mathrm{PGL}_{3}(q)$ is a semisimple element of $\mathrm{PGL}_{3}(q)$, which we denote by $\bar{s}$. Since $\tau$ is the inverse-transpose, the eigenvalues of $\tau(\bar{s})$ are exactly those of $\bar{s}^{-1}$. Therefore, $\bar{s}^{-1}$ and $\tau(\bar{s})$ are conjugates in $\mathrm{PGL}_{3}(q)$. This and [20, Corollary 2.5] imply that

$$
\left(\chi_{\bar{s}}\right)^{\tau}=\chi_{\tau(\bar{s})}=\chi_{\bar{s}^{-1}}=\overline{\chi_{\bar{s}}} .
$$

On the other hand, as the eigenvalues of $\bar{s}$ and $\bar{s}^{-1}$ are different, the semisimple character $\chi_{s}$ is non-real, which means that

$$
\overline{\chi_{\bar{s}}} \neq \chi_{\bar{s}} .
$$


Therefore, we have shown that $\chi_{\bar{s}} \in \operatorname{Irr}\left(\mathrm{SL}_{3}(q)\right)$ is not invariant under $\tau$. Moreover, since $\bar{s}$ is contained in $\left[\mathrm{PGL}_{3}(q), \mathrm{PGL}_{3}(q)\right]=\mathrm{PSL}_{3}(q), \mathbf{Z}\left(\mathrm{SL}_{3}(q)\right) \subseteq$ $\operatorname{Ker}\left(\chi_{\bar{s}}\right)$ so that $\chi_{\bar{s}}$ can also be viewed as a character of $\operatorname{PSL}_{3}(q)$.

We have produced the irreducible character $\chi_{\bar{s}} \in \operatorname{Irr}\left(G^{\prime}\right)$ which is not invariant under $\tau$. It is easy to calculate that $\chi_{\bar{s}}(1)=(q-1)^{2}(q+1)$. Since $\mathrm{PGL}_{3}(q)$ has no irreducible character of degree $3 \chi_{\bar{s}}(1), \chi_{\bar{s}}$ is invariant under $d$. It follows that $\chi_{\bar{s}}$ is not $\alpha$-invariant, which implies that $G / C$ has a character degree which is a proper multiple of $\chi_{\bar{s}}(1)=(q-1)^{2}(q+1)$. This is impossible.

(3ii) Assume $\alpha=d^{a} \sigma^{b} \tau^{c}$ with $0<b<f, 0 \leq a<3$, and $0 \leq c<2$. Now we can find $\omega_{1} \in \overline{\mathbb{F}_{p}}$ of order $q+1$. We then consider a semisimple element $s_{1} \in \mathrm{SL}_{3}(q)$ with eigenvalues $1, \omega_{1}$, and $\omega_{1}^{q}=\omega_{1}^{-1}$. The image of $s_{1}$ under the projection $\mathrm{GL}_{3}(q) \rightarrow \mathrm{PGL}_{3}(q)$ is a semisimple element of $\operatorname{PGL}_{3}(q)$, which we denote by $\overline{s_{1}}$. Now the eigenvalues of $\overline{s_{1}}, \overline{s_{1}}-1$, and $\tau\left(\overline{s_{1}}\right)$ are all the same. Therefore, by [20, Corollary 2.5],

$$
\left(\chi_{\overline{s_{1}}}\right)^{\tau}=\chi_{\tau\left(\overline{s_{1}}\right)}=\chi_{\overline{s_{1}}-1}=\overline{\chi_{\overline{s_{1}}}}=\chi_{\overline{s_{1}}} .
$$

In other words, $\chi_{\overline{s_{1}}}$ is fixed under $\tau$. As above, since $\overline{s_{1}} \in\left[\mathrm{PGL}_{3}(q), \mathrm{PGL}_{3}(q)\right]$, we have $\mathbf{Z}\left(\mathrm{SL}_{3}(q)\right) \subseteq \operatorname{Ker}\left(\chi_{\overline{s_{1}}}\right)$ so that $\chi_{\overline{s_{1}}}$ can also be viewed as a character of $\operatorname{PSL}_{3}(q)$. So we found the character $\chi_{\overline{s_{1}}} \in \operatorname{Irr}\left(G^{\prime}\right)$ of degree $\chi_{\overline{s_{1}}}(1)=q^{3}-1$ which is fixed under $\tau$. Since $\mathrm{PGL}_{3}(q)$ has no irreducible character of degree $3 \chi_{\overline{s_{1}}}(1), \chi_{\overline{s_{1}}}$ is also fixed under $d$.

Now the eigenvalues of $\sigma^{b}\left(\overline{s_{1}}\right)$ are $1, \omega^{p^{b}}$, and $\omega^{q p^{b}}$, which are not the same as those of $\overline{s_{1}}$. Using [20, Corollary 2.5] again, we deduce that $\chi_{\overline{s_{1}}} \in \operatorname{Irr}\left(G^{\prime}\right)$ is not fixed under $\sigma^{b}$. It follows that $\chi_{\overline{s_{1}}}$ is not $\alpha$-invariant. Therefore, $G$ has a character degree which is a proper multiple of $\chi_{\overline{s_{1}}}(1)=q^{3}-1$, a contradiction again.

(3iii) Finally, assume $G / C$ induces only the diagonal automorphisms on $G^{\prime}$. Then $G / C \cong \mathrm{PGL}_{3}(q)$. From [7] again, $\mathrm{SL}_{3}(q)$ has exactly three (nonfaithful) irreducible characters of degree $(q+1)\left(q^{2}+q+1\right) / 3$ which are fused under the diagonal automorphisms. Therefore, if $\chi$ is an irreducible character of degree $(q+1)\left(q^{2}+q+1\right) / 3$ of $G, \chi$ must lie above $\mu \in \operatorname{Irr}\left(G^{\prime}\right)$ with $\mu(1)<$ $(q+1)\left(q^{2}+q+1\right) / 3$. This cannot happen since $\mathrm{SL}_{3}(q)$ has no character degree which is nontrivial proper divisor of $(q+1)\left(q^{2}+q+1\right) / 3$.

\section{Step 5 for $\mathrm{SL}_{3}(q)$}

We have shown that $G=G^{\prime} \circ C$ where $C:=\mathbf{C}_{G}\left(G^{\prime}\right)$ is Abelian and $G^{\prime} \cong \mathrm{SL}_{3}(q)$ or $\operatorname{PSL}_{3}(q)$. We need to show that $G^{\prime} \cong \operatorname{SL}_{3}(q)$. Suppose by contradiction that $G^{\prime} \cong \operatorname{PSL}_{3}(q)$. Then $G=G^{\prime} \times C$, and thus $\operatorname{cd}(G)=\operatorname{cd}\left(G^{\prime}\right)$, which means that $\operatorname{cd}\left(\mathrm{SL}_{3}(q)\right)=\operatorname{cd}\left(\mathrm{PSL}_{3}(q)\right)$. This is impossible since

$$
\frac{1}{3}(q-1)^{2}(q+1) \in \operatorname{cd}\left(\mathrm{SL}_{3}(q)\right)-\operatorname{cd}\left(\operatorname{PSL}_{3}(q)\right),
$$


and we have completed the proof of Theorem 4.1.

\section{REFERENCES}

[1] C. Bessenrodt, H. N. Nguyen, J. B. Olsson and H. P. Tong-Viet, Complex group algebras of the double covers of the symmetric and alternating groups, Algebra Number Theory 9 (2015), 601-628. MR 3340546

[2] M. Bianchi, D. Chillag, M. L. Lewis and E. Pacifici, Character degree graphs that are complete graphs, Proc. Amer. Math. Soc. 135 (2007), 671-676. MR 2262862

[3] T. Breuer, Manual for the GAP character table library, Version 1.1, RWTH Aachen, 2004.

[4] R. W. Carter, Finite groups of Lie type. Conjugacy classes and complex characters, Wiley, New York, 1985. MR 0794307

[5] J. H. Conway, R. T. Curtis, S. P. Norton, R. A. Parker and R. A. Wilson, Atlas of finite groups, Oxford University Press, London, 1984. MR 0827219

[6] P. Erdös and J. L. Selfridge, The product of consecutive integers is never a power, Illinois J. Math. 19 (1975), 292-301. MR 0376517

[7] J. S. Frame and W. A. Simpson, The character tables for $\mathrm{SL}_{3}(q), \mathrm{SU}_{3}(q), \operatorname{PSL}_{3}(q)$, $\mathrm{PSU}_{3}\left(q^{2}\right)$, Canad. J. Math. 25 (1973), 486-494. MR 0335618

[8] D. Gorenstein, R. Lyons and R. Solomon, The classification of the finite simple groups, Number 3, Mathematical Surveys and Monographs, American Mathematical Society, Providence, RI, 1998. MR 1490581

[9] B. Huppert, Some simple groups which are determined by the set of their character degrees I, Illinois J. Math. 44 (2000), 828-842. MR 1804317

[10] I. M. Isaacs, Character theory of finite groups, Dover Publications, New York, 1994. MR 1280461

[11] I. M. Isaacs, G. Malle and G. Navarro, A reduction theorem for the McKay conjecture, Invent. Math. 170 (2007), 33-101. MR 2336079

[12] O. H. King, The subgroup structure of finite classical groups in terms of geometric configurations, London. Math. Soc. Lecture Note Series 327 (2005), 29-56. MR 2187733

[13] M. Lewis and D. White, Connectedness of degree graphs of non-solvable groups, J. Algebra 266 (2003), 51-76. MR 1994528

[14] G. Malle, Extensions of unipotent characters and the inductive McKay condition, J. Algebra 320 (2008), 2963-2980. MR 2442005

[15] A. Moretó, An answer to a question of Isaacs on character degree graphs, Adv. Math. 201 (2006), 90-101. MR 2204750

[16] A. Moretó, Complex group algebras of finite groups: Brauer's problem 1, Adv. Math. 208 (2007), 236-248. MR 2304316

[17] T. Nagell, Des équations indéterminées $x^{2}+x+1=y^{n}$ et $x^{2}+x+1=3 y^{n}$, Norsk. mat. Foren. Skr. Serie I (1921), 1-14.

[18] G. Navarro, The set of character degrees of a finite group does not determine its solvability, Proc. Amer. Math. Soc. 143 (2015), 989-990. MR 3293716

[19] G. Navarro and N. Rizo, Nilpotent and perfect groups with the same set of character degrees, J. Algebra Appl. 13 (2014), 1450061. 3 pp. MR 3225128

[20] G. Navarro, P. H. Tiep and A. Turull, Brauer characters with cyclotomic field of values, J. Pure Appl. Algebra 212 (2008), 628-635. MR 2365337

[21] H. N. Nguyen, Quasisimple classical groups and their complex group algebras, Israel J. Math. 195 (2013), 973-998. MR 3096579

[22] H. N. Nguyen and H. P. Tong-Viet, Characterizing finite quasisimple groups by their complex group algebras, Algebr. Represent. Theory 17 (2014), 305-320. MR 3160726

[23] H. N. Nguyen and H. P. Tong-Viet, Recognition of finite quasi-simple groups by the degrees of their irreducible representations, Groups St Andrews 2013, London Math. 
Soc. Lecture Note Ser., vol. 422, Cambridge Univ. Press, Cambridge, 2015, pp. 439456. MR 3495673

[24] H. N. Nguyen, H. P. Tong-Viet and T. P. Wakefield, Projective special linear groups $\mathrm{PSL}_{4}(q)$ are determined by the set of their character degrees, J. Algebra Appl. 11 (2012), 1250108. 26 pp. MR 2997450

[25] H. P. Tong-Viet, The simple Ree groups ${ }^{2} \mathrm{~F}_{4}\left(q^{2}\right)$ are determined by the set of the character degrees, J. Algebra 339 (2011), 357-369. MR 2811327

[26] H. P. Tong-Viet and T. P. Wakefield, On Huppert's conjecture for $\mathrm{G}_{2}(q), q \geq 7$, J. Pure Appl. Algebra 216 (2012), 2720-2729. MR 2943753

[27] H. P. Tong-Viet and T. P. Wakefield, On Huppert's conjecture for ${ }^{3} \mathrm{D}_{4}(q), q \geq 3$, Algebr. Represent. Theory 16 (2013), 470-490. MR 3036002

[28] T. P. Wakefield, Verifying Huppert's conjecture for the simple groups of Lie type of rank two, Ph.D. thesis, Kent State University, 2008. MR 2711647

[29] T. P. Wakefield, Verifying Huppert's conjecture for $\operatorname{PSL}_{3}(q)$ and $\operatorname{PSU}_{3}\left(q^{2}\right)$, Comm. Algebra 37 (2009), 2887-2906. MR 2543522

[30] D. L. White, Character degrees of extensions of $\mathrm{PSL}_{2}(q)$ and $\mathrm{SL}_{2}(q)$, J. Group Theory 16 (2013), 1-33. MR 3008309

[31] K. Zsigmondy, Zur theorie der Potenzreste, Monath. Math. Phys. 3 (1892), 265-284. MR 1546236

Nguyen Ngoc Hung, Department of Mathematics, The University of Akron, AKron, OH 44325, USA

E-mail address: ungnguyen@uakron.edu

Philani R. Majozi, Faculty of Science and Agriculture, Department of Mathematical Sciences, University of Zululand, KwaDlangezwa 3880, South Africa

E-mail address: majozip@unizulu.ac.za

Hung P. Tong-Viet, Department of Mathematical Sciences, Kent State UniVERSity, KENT, OH 44242, USA

E-mail address: htongvie@math.kent.edu

Thomas P. Wakefield, Department of Mathematics and Statistics, Youngstown State University, One University Plaza, Youngstown, OH 44555, USA

E-mail address: tpwakefield@ysu.edu 